TOPICS IN ALGEBRA

BANACH CENTER PUBLICATIONS, VOLUME 26. PART I

PWN-POLISH SCIENTIFIC PUBLISHFER

WARSAW 1990

\title{
COMPUTATIONAL TECHNIQUES FOR PI-ALGEBRAS
}

\author{
VESSELIN DRENSKY \\ Institute of Mathematics, Bulgarian Academy of Sciences \\ Sofia, Bulgaria
}

\begin{abstract}
Introduction
Let $R$ be a (not necessarily associative) algebra over a field of characteristic 0 and let $R$ satisfy a polynomial identity, i.e. $R$ is a PI-algebra. When studying the properties of $R$, the following natural question arises: How many identities of $R$ are there? Of course, we have to decide how to measure the quantity of the polynomial identities. There are some numerical invariants of $R$ as the codimension and cocharacter sequences and the Hilbert series of the T-ideal of $R$ and they are objects of intensive investigation.

The main purpose of this paper is to survey and present from a unique point of view some recent quantitative results on PI-algebras. Using the powerful technique of the representation theory of the symmetric and general linear groups we present effective computing methods for concrete PI-algebras. In particular, we apply these methods for studying the polynomial identities of some important associative, Lie and Jordan algebras.

The paper is organized as follows. Section 1 gives the necessary background on PI-algebras and representation theory of the symmetric and general linear groups. Sections 2 is devoted to the free Lie and commutative algebras, some other relatively free algebras and to an important reduction of the computations in the case of varieties of unitary algebras. Section 3 studies the polynomial identities of algebras which in some sense are similar to the $2 \times 2$ matrix algebra. Section 4 handles the calculation of the codimensions of $T$-ideals and the Hilbert series of relatively free algebras. For other applications of the presented computational techniques we refer to the surveys $[5,21,22]$.
\end{abstract}

\section{The background}

\subsection{Varieties of algebras}

We fix some notation: $K$ is a field of characteristic $0, X=\left\{x_{1}, x_{2}, \ldots\right\}$, $F=K\{X\}$ is the absolutely free algebra of infinite rank. The elements of $F$ are

2 Banach Center t. 26, cz. I

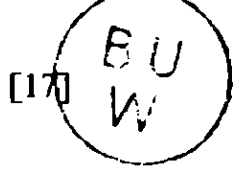


polynomials in noncommutative and nonassociative variables and with zero constant terms. Usually we consider the products left-normed; hence $x_{1} x_{2} x_{3}=\left(x_{1} x_{2}\right) x_{3}$.

The element $f\left(x_{1}, \ldots, x_{n}\right) \in K\{X\}$ is called a polynomial identity for the $K$-algebra $R$ if $f\left(r_{1}, \ldots, r_{n}\right)=0$ for all $r_{1}, \ldots, r_{n} \in R$. The class $U$ of all algebras satisfying a given system of identities $\left\{u_{i}\left(x_{1}, \ldots, x_{n_{i}}\right) \mid i \in I\right\}$ is called a variety of algebras. The set of all polynomial identities $U$ satisfied by the variety $U$ (respectively by the algebra $R$ ) is a two-sided ideal of $K\{X\}$ called a T-ideal and denoted by $T(U)$ (respectively $T(R)$ ). We use the same letters $U$ and $U$ respectively for the $T$-ideals and the related varieties. The algebra $F(U)=K\{X\} / T(U)$ (with the same set of generators $X=\left\{x_{1}, x_{2}, \ldots\right\}$ ) is the relatively free algebra of $\boldsymbol{U}$. We denote by $F_{m}(\boldsymbol{U})$ the subalgebra of $F(\boldsymbol{U})$ generated by the subset $\left\{x_{1}, \ldots, x_{m}\right\}$. Moreover, for a subspace $Q$ of $F$ we denote by $Q(U)$ the image of $Q$ under the canonical homomorphism $F \rightarrow F / U=F(U)$.

1.1.1. ExAmples. The class of all associative algebras forms a variety defined by the identity $\left(x_{1} x_{2}\right) x_{3}-x_{1}\left(x_{2} x_{3}\right)$; the varieties of all Lie and all Jordan algebras are determined by the sets of identities $\left\{x_{1}^{2},\left(x_{1}, x_{2}\right) x_{3}\right.$ $\left.+\left(x_{2} x_{3}\right) x_{1}+\left(x_{3} x_{1}\right) x_{2}\right\}$ and $\left\{x_{1} x_{2}-x_{2} x_{1},\left(x_{2} x_{1}\right)\left(x_{1} x_{1}\right)-\left(x_{2}\left(x_{1} x_{1}\right)\right) x_{1}\right\}$, respectively, etc.

We denote by $P_{n}$ the vector space of all multilinear polynomials in $K\{X\}, P_{n}=\left\{\sum a_{\sigma d}\left(x_{\sigma(1)} \ldots\right)\left(\ldots x_{\sigma(n)}\right) \mid a_{\sigma d} \in K\right\}$, where the summation runs over all permutations $\sigma$ of the symmetric group $\operatorname{Sym}(n)$ acting on $\{1, \ldots, n\}$ and over all distributions $d$ of brackets. It is well known that every variety $U$ can be determined by its multilinear identities. Let $P_{n}(U)=P_{n} /\left(P_{n} \cap T(U)\right)$, $n=1,2, \ldots$ The sequence of codimensions of the variety $U$ (or of the T-ideal $U$ ) is defined by $c_{n}(U)=c_{n}(U)=\operatorname{dim} P_{n}(U), n=1,2, \ldots$ Additionally, the generating function $c(U, t)=\sum c_{n}(U) t^{n}$ and the exponential generating function $\tilde{c}(\boldsymbol{U}, t)=\sum c_{n}(\boldsymbol{U}) t^{n} / n$ ! are called the codimension series and the exponential codimension series of $U$, respectively.

The relatively free algebra of rank $m$ is a graded vector space, $F_{m}(\boldsymbol{U})=$ $\sum F_{m}^{(n)}(\boldsymbol{U})$, where $F_{m}^{(n)}(\boldsymbol{U})$ is the homogeneous component of degree $n$. The Hilbert series of $F_{m}(U)$ is defined by $H_{m}(U, t)=H\left(F_{m u}(U), t\right)=\sum \operatorname{dim} F_{m}^{(n)}(\boldsymbol{U}) t^{n}$; $F_{m}(U)$ has another grading counting the degree in any variable and the corresponding Hilbert series is

$$
H\left(\boldsymbol{U}, t_{1}, \ldots, t_{m}\right)=\sum \operatorname{dim} F_{m}^{\left(n_{1}, \ldots, n_{m}\right)}(U) t_{t_{1}}^{n_{1}} \ldots t_{m}^{n_{m}}
$$

Clearly $H_{m}(\boldsymbol{U}, t)=H(\boldsymbol{U}, t, \ldots, t)$ (with $m$ 's).

1.1.2. Proposition. Let $U$ be a T-ideal in $K\{X\}$ and let $\tilde{K}$ be an extension of the base field $K$. Then $\tilde{F}(\tilde{U})=\tilde{K} \otimes_{K} F(U)$ is the relatively free algebra of the variety $\boldsymbol{U}$ of $\tilde{K}$-algebras defined by the same system $U$ of polynomial identities (assuming that $K\{X\}$ is canonically embedded into $\widetilde{K}\{X\}=\widetilde{K} \otimes_{K} K\{X\}$ ). 
This proposition allows one to extend the base field and, if necessary, consider $K$ algebraically closed.

\subsection{Representations of $\operatorname{Sym}(n)$ and $\mathrm{GL}_{m}$}

The vector space $P_{n}$ has a natural structure of a left $\operatorname{Sym}(n)$-module defined by

$$
\sigma:\left(x_{i_{1}} \ldots\right)\left(\ldots x_{i_{n}}\right) \rightarrow\left(x_{\sigma\left(i_{1}\right)} \ldots\right)\left(\ldots x_{\sigma\left(i_{n}\right)}\right), \quad \sigma \in \operatorname{Sym}(n),
$$

and for a long period this was the main tool for quantitative investigation of the polynomial identities (see $[54,57,25]$ for details). It is known that the representations of $\operatorname{Sym}(n)$ are related to the polynomial representations of the general linear group $\mathrm{GL}_{m}$. The canonical action of $\mathrm{GL}_{m}$ on the $m$-dimensional vector space spanned by $x_{1}, \ldots, x_{m}$ can be extended diagonally to $F_{m}=K\left\{x_{1}, \ldots, x_{m}\right\}$ by

$$
g:\left(x_{i_{1}} \ldots\right)\left(\ldots x_{i_{n}}\right) \rightarrow\left(g\left(x_{i_{1}}\right) \ldots\right)\left(\ldots g\left(x_{i_{n}}\right)\right), \quad g \in \mathrm{GL}_{m} .
$$

Although this action was used incidentally before 1980 , its systematical application began in $[5,10]$ (see also [26] for the formalism of the equivalence of the application of $\operatorname{Sym}(n)$ and $\mathrm{GL}_{m}$ ).

The irreducible representations of $\operatorname{Sym}(n)$ and $\mathrm{GL}_{m}$ are described by partitions and Young diagrams $[31,65]$. For a partition $\lambda=\left(\lambda_{1}, \ldots, \lambda_{r}\right)$ of $n$, $\hat{\lambda}_{1} \geqslant \ldots \geqslant \lambda_{r} \geqslant 0, \lambda_{1}+\ldots+\lambda_{r}=n$, we consider the corresponding Young diagram $[\lambda]$, the number $n=\|\lambda\|$ of boxes of $[\lambda]$ and the related irreducible $\operatorname{Sym}(n)$-module $M(\lambda)$ and $\mathrm{GL}_{m}$-module $N_{m}(\lambda)$. In order to obtain generators for the irreducible submodules of $P_{n}$ and $F_{m}$ we use the following device:

(i) As a $\mathrm{GL}_{m}$-module, the homogeneous component $F_{m}^{(n)}$ is a direct sum of the $\mathrm{GL}_{m}$-submodules $N_{m, d}^{(n)}$, where $d$ is a fixed distribution of brackets and $N_{m, d}^{(n)} \stackrel{\mathscr{L}}{=} A_{m}^{(n)}, A_{m}=K\left\langle x_{1}, \ldots, x_{m}\right\rangle$ being the free associative algebra. Here the $\mathrm{GL}_{m}$-module isomorphism $\varphi$ deletes the brackets in $N_{m, d}^{(n)}$.

(ii) First, we determine a generator for the irreducible submodules of $A_{m}^{(n)}$. We define an action of $\operatorname{Sym}(n)$ on $A_{m}^{(n)}$ by

$$
\left(x_{i_{1}} \ldots x_{i_{n}}\right) \varrho^{-1}=x_{i_{Q^{(1)}}} \ldots x_{i_{Q^{(n)}}}, \quad \varrho \in \operatorname{Sym}(n) .
$$

Then every submodule $N_{m}(\lambda)$ of $A_{m}^{(n)}$ is generated by a nonzero element

$$
f\left(x_{1}, \ldots, x_{r}\right)=\prod S_{r_{i}}\left(x_{1}, \ldots, x_{r_{i}}\right) \sum a_{\varrho} \varrho,
$$

where $a_{e} \in K, \varrho$, runs over $\operatorname{Sym}(n), S_{p}\left(x_{1}, \ldots, x_{p}\right)=\sum(\operatorname{sign} \sigma) x_{\sigma(1)} \ldots x_{\sigma(p)}$ is the standard polynomial and $r_{1}, \ldots, r_{k}$ are the lengths of the columns of the diagram $[\lambda]$.

(iii) An arbitrary irreducible $\mathrm{GL}_{m}$-submodule $N_{m}(\lambda)$ of $F_{m}$ is generated by an element

$$
\sum f_{d}\left(x_{1}, \ldots, x_{r}\right),
$$

where $f_{d}$ is of the form (1) and the summation runs over all distributions of brackets. We call $\left(1^{\prime}\right)$ a standard generator of $N_{m}(\lambda)$. It follows from the 
representation theory of $\mathrm{GL}_{m}$ that the standard generator is uniquely determined up to a multiplicative constant.

(iv) A generator of the irreducible $\operatorname{Sym}(n)$-submodule $M(\lambda)$ of $P_{n}$ can be obtained by a linearization of a suitable standard generator of $N_{m}(\hat{\lambda})$.

For an arbitrary $T$-ideal $U$ of $F$, the subspaces $U \cap P_{n}$ and $U \cap F_{m}$ are $\operatorname{Sym}(n)$ - and $\mathrm{GL}_{m}$-modules, respectively. Hence the relatively free algebra $F(U)$ inherits the actions of $\operatorname{Sym}(n)$ and $\mathrm{GL}_{m}$. In particular, the $\operatorname{Sym}(n)$-character sequence $\chi\left(P_{n}(U)\right), n=1,2, \ldots$, is called the cocharacter sequence of $U$. The following assertion gives the equivalence of the application of the representation theory of $\operatorname{Sym}(n)$ and $\mathrm{GL}_{m}$.

1.2.1. Theorem $[5,10]$. (i) Let $\Lambda$ and $\Lambda^{*}$ be the lattices of submodules of $F_{m}^{(n)}$ and $P_{n}$, respectively (with respect to the sum and intersection of submodules). Then there is a lattice monomorphism $\psi: \Lambda \rightarrow \Lambda^{*}$ such that $\psi\left(N_{m}(\lambda)\right)=M(\lambda)$ and a generator of $M(\lambda)$ is obtained by a linearization of the standard generator of $N_{m}(\lambda)$. The image of $\psi(\Lambda)$ coincides with $\sum M(\lambda)$, where the diagrams $[\lambda]$ have at most $m$ rows. In particular, $\psi$ is an isomorphism for $m \geqslant n$.

(ii) For every variety $U, P_{n}(U)$ and $F_{m}^{(n)}(U)$ have the same module structure: If $P_{n}(U)=\sum k(\lambda) M(\lambda)$, then $F_{m}^{(n)}(U)=\sum k(\lambda) N_{m}(\lambda)$.

It turns out that for concrete computations the representations of $\mathrm{GL}_{m}$ are more convenient than those of $\operatorname{Sym}(n)$. For example, the polynomial identities $h\left(x_{1}, \ldots, x_{n}\right)=\sum x_{\sigma(1)} \ldots x_{\sigma(n)}$ and $x_{1}^{n}$ from $A_{n}^{(n)}$ are equivalent and generate $M(n)$ and $N_{n}(n)$ respectively but $x_{1}^{n}$ is written more compactly. Additionally, the representations of $\mathrm{GL}_{m}$ allow the classical invariant theory to be applied to PI-algebras (see e.g. $[26,50]$ ).

For a multihomogeneous polynomial $f\left(x_{1}, \ldots, x_{m}\right)$ we denote by

$$
f\left(x_{1}, y_{11}, \ldots, y_{1 n_{1}}|\ldots| x_{m}, y_{m 1}, \ldots, y_{m n_{m}}\right)
$$

the partial linearization of $f\left(x_{1}, \ldots, x_{m}\right)$ which equals the component of $f\left(x_{1}+y_{11}+\ldots+y_{1 n_{1}}, \ldots, x_{m}+y_{m 1}+\ldots+y_{m n_{m}}\right)$ multilinear in $y_{i j}$. The following result allows one to find standard generators of $N_{m}(\lambda)$.

1.2.2. ThEOREM [36]. Let $\lambda=\left(\lambda_{1}, \ldots, \lambda_{r}\right)$ be a partition and let $f\left(x_{1}, \ldots, x_{r}\right)$ be a nonzero polynomial from $F_{m}$ which is homogeneous of degree $\lambda_{i}$ in $x_{i}, i=1, \ldots, r$. Then $f$ is a standard generator of $N_{m}(\lambda)$ if and only if $f\left(x_{1}|\ldots| x_{j}, x_{i}|\ldots| x_{r}\right)=0$ for every pair $(i, j), 1 \leqslant i<j \leqslant r$.

In the representation theory of $\mathrm{GL}_{m}$, the role of characters of $\mathrm{GL}_{m}$ is played by the Schur functions $S_{\lambda}\left(t_{1}, \ldots, t_{m}\right)$ [41]. They are symmetric polynomials from $K\left[t_{1}, \ldots, t_{m}\right]$ and

$$
S_{\lambda}\left(t_{1}, \ldots, t_{m}\right)=\sum a_{i} t_{1}^{i_{1}} \ldots t_{m}^{i_{m}}=H\left(N_{m}(\lambda), t_{1}, \ldots, t_{m}\right), \quad N_{m}(\lambda) \subset A_{m}^{(n)} .
$$

The coefficient $a_{i}$ equals the number of semistandard $\lambda$-tableaux with content $i=\left(i_{1}, \ldots, i_{m}\right)$, i.e. the Schur functions can be obtained in a combinatorial way 
(see Section 1.3). It is known that the Hilbert series of a $\mathrm{GL}_{m}$-module determines the module uniquely. In particular, $H\left(F_{m}(U), t_{1}, \ldots, t_{m}\right)$ $=\sum k(\lambda) S_{\lambda}\left(t_{1}, \ldots, t_{m}\right)$ if and only if $F_{m}(U)=\sum k(\lambda) N_{m}(\lambda)$.

\subsection{The Littlewood-Richardson rule}

1.3.1. Definition [41]. Let $\lambda=\left(\lambda_{1}, \ldots, \lambda_{r}\right), \mu=\left(\mu_{1}, \ldots, \mu_{s}\right)$ and $v=\left(v_{1}, \ldots, v_{r}\right)$ be partitions, $v_{i} \geqslant \lambda_{i}$ and $\|v\|=\|\lambda\|+\|\mu\|$.

(i) A diagram of shape $[v-\lambda]$ is a scheme of boxes obtained from the diagram $[\nu]$ by removing the boxes of the diagram $[\lambda]$. When $\|\lambda\|=0$, $[v-\lambda]=[v]$.

(ii) A $[v-\lambda]$-tableau with content $\mu$ is the diagram $[v-\lambda]$ whose boxes are filled in with $\mu_{1}$ numbers $1, \ldots, \mu_{s}$ numbers $s$.

(iii) A tableau is semistandard if its entries do not decrease from left to right in the rows and increase from top to bottom in the columns.

(iv) The sequence $w(T)$ is obtained from a tableau $T$ by listing the entries of $T$ from right to left, consecutively reading the rows from top to bottom (as in Arabic).

(v) The sequence $w=a_{1}, a_{2}, \ldots, a_{n}$ is a lattice permutation if it contains the symbols $1,2, \ldots, s$ and for each $1 \leqslant k \leqslant n$ and $1 \leqslant i \leqslant s-1$, the number $i$ occurs in $a_{1}, \ldots, a_{k}$ no less than $i+1$ times.

13.2. THEOREM (The Littlewood-Richardson rule). The following isomorphism of $\mathrm{GL}_{m}$-modules holds:

$$
N_{m}(\lambda) \otimes_{K} N_{m}(\mu)=\sum_{v} c_{\lambda \mu}^{v} N_{m}(v)
$$

where $c_{\lambda \mu}^{\nu}$ is the number of semistandard tableaux Tof shape $[\nu-\lambda]$ with content $\mu$, such that the sequence $w(T)$ is a lattice permutation.

An important role in our concrete computations is played by the following consequence of Theorem 1.3.2.

1.3.3. CoRollary. $N_{m}\left(\lambda_{1}, \ldots, \lambda_{m}\right) \otimes_{K} N_{m}(s) \cong \sum N_{m}\left(\mu_{1}, \ldots, \mu_{m}\right)$, where $\|\mu\|=\|\lambda\|+s$ and $\mu_{1} \geqslant \lambda_{1} \geqslant \mu_{2} \geqslant \lambda_{2} \geqslant \ldots \geqslant \mu_{m} \geqslant \lambda_{m}$.

In particular, for $s=1$ the corollary coincides with the branching theorem.

1.3.4. Rule. The following device allows us to obtain the standard generators of the tensor product of $\mathrm{GL}_{m}$-modules. It is a combination of Theorems 1.2.2 and 1.3.2. Its preliminary version has been used in $[14,36]$. In order to simplify the rule, we consider $N_{m}(\lambda)$ and $N_{m}(\mu)$ as submodules of $A_{m}^{(p)}$ and $A_{m}^{(q)}$, respectively, where $p=\|\lambda\|$ and $q=\|\mu\|$, and identify $A_{m}^{(p)} \otimes_{\mathrm{K}} A_{m}^{(q)}$ with $A_{m}^{(p+q)}$. Additionally, we fix partitions $\lambda, \mu, v$ as in the Littlewood-Richardson rule, and $f_{\lambda}\left(x_{1}, \ldots, x_{m}\right)$ and $f_{\mu}\left(x_{1}, \ldots, x_{m}\right)$ are the standard generators of $N_{m}(\lambda)$ and $N_{m}(\mu)$, respectively. 
(i) Find all semistandard tableaux $T_{\lambda}(\alpha)$ and $T_{\mu}(\beta)$ with contents $\alpha=\left(\alpha_{1}, \ldots, \alpha_{m}\right), \beta=\left(\beta_{1}, \ldots, \beta_{m}\right)$, respectively, $\alpha_{i}+\beta_{i}=v_{i}$.

(ii) For every tableau $T_{\lambda}(\alpha)$ let

$$
f_{\lambda \alpha}=f_{\lambda}\left(x_{11}, \ldots, x_{1 \lambda_{1}}|\ldots| x_{m 1}, \ldots, x_{m \lambda_{m}}\right)
$$

be the linearization of $f_{\lambda}$, where $x_{i j}=x_{k}, k$ being the $(i, j)$-entry of $T_{\lambda}(\alpha)$, and similarly for $f_{\mu}$ and $T_{\mu}(\beta)$.

(iii) Write $f\left(x_{1}, \ldots, x_{m}\right)=\sum a_{\alpha \beta} f_{\lambda \alpha} f_{\mu \beta}$ with unknown $a_{\alpha \beta} \in K$.

(iv) Assuming that $f\left(x_{1}|\ldots| x_{j}, x_{i}|\ldots| x_{m}\right)=0$ for all pairs $(i, j)$, $1 \leqslant i<j \leqslant m$, obtain a linear homogeneous system for $a_{\alpha \beta}$. Any nonzero solution of this system gives a standard generator for $N_{m}(v) \subset N_{m}(\lambda) \otimes_{K} N_{m}(\mu)$.

By using additional information, this rule can be simplified for concrete cases.

1.3.5. EXAMPLE. Let $\lambda=(2,1), \mu=(2), v=\left(3,1^{2}\right), f_{\lambda}\left(x_{1}, x_{2}\right)=S_{2}\left(x_{1}, x_{2}\right) x_{1}$ $=\left(x_{1} x_{2}-x_{2} x_{1}\right) x_{1}=\left[x_{1}, x_{2}\right] x_{1}, f_{\mu}\left(x_{1}\right)=x_{1}^{2}$. Then

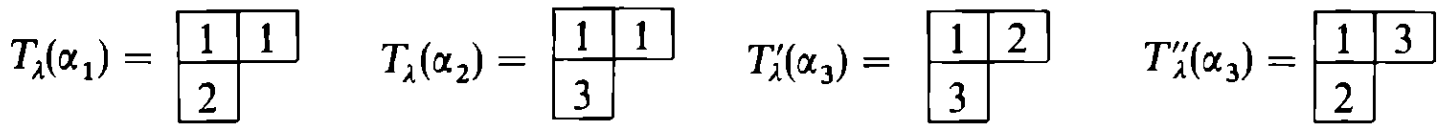

$$
\begin{aligned}
& T_{\mu}\left(\beta_{1}\right)=\begin{array}{l|l|l|l|l|l|l|l|l|l|l|l|l|l|l|}
\hline 1 & 3 & T_{\mu}\left(\beta_{2}\right)=1 & 2 \\
\hline
\end{array} \\
& f_{\lambda \alpha_{1}}=f_{\lambda}\left(x_{1}, x_{1} \mid x_{2}\right)=2 f_{\lambda}\left(x_{1}, x_{2}\right), \quad f_{\mu \beta_{1}}=f_{\mu}\left(x_{1}, x_{3}\right)=x_{1} x_{3}+x_{3} x_{1}, \\
& f_{\lambda \alpha_{2}}=2 f_{\lambda}\left(x_{1}, x_{3}\right), \quad f_{\mu \beta_{2}}=x_{1} x_{2}+x_{2} x_{1}, \\
& f_{\lambda x_{3}}^{\prime}=\left[x_{1}, x_{3}\right] x_{2}+\left[x_{2}, x_{3}\right] x_{1}, f_{\lambda a_{3}}^{\prime \prime}=\left[x_{1}, x_{2}\right] x_{3}+\left[x_{3}, x_{2}\right] x_{1}, f_{\mu \rho_{3}}=2 x_{1}^{2} \text {, } \\
& f\left(x_{1}, x_{2}, x_{3}\right)=2\left\{a_{1}\left[x_{1}, x_{2}\right] x_{1}\left(x_{1} x_{3}+x_{3} x_{1}\right)+a_{2}\left[x_{1}, x_{3}\right] x_{1}\left(x_{1} x_{2}+x_{2} x_{1}\right)\right. \\
& \left.+a_{3}^{\prime}\left(\left[x_{1}, x_{3}\right] x_{2}+\left[x_{2}, x_{3}\right] x_{1}\right) x_{1}^{2}+a_{3}^{\prime \prime}\left(\left[x_{1}, x_{2}\right] x_{3}+\left[x_{3}, x_{2}\right] x_{1}\right) x_{1}^{2}\right\} \text {, } \\
& f\left(x_{1}\left|x_{2}, x_{1}\right| x_{3}\right)=f\left(x_{1}, x_{1}, x_{3}\right)=2\left(2 a_{2}+2 a_{3}^{\prime}-a_{3}^{\prime \prime}\right)\left[x_{1}, x_{3}\right] x_{1}^{3}=0 \text {, } \\
& f\left(x_{1}\left|x_{2}\right| x_{3}, x_{1}\right)=2\left(2 a_{1}-a_{3}^{\prime}+2 a_{3}^{\prime \prime}\right)\left[x_{1}, x_{2}\right] x_{1}^{3}=0 \text {, } \\
& f\left(x_{1}\left|x_{2}\right| x_{3}, x_{2}\right)=2\left[x_{1}, x_{2}\right]\left\{\left(a_{1}+a_{2}\right) x_{1}\left(x_{1} x_{2}+x_{2} x_{1}\right)+\left(a_{3}^{\prime}+a_{3}^{\prime \prime}\right) x_{2} x_{1}^{2}\right\}=0 \text {. }
\end{aligned}
$$

Therefore we obtain the system

$$
\begin{aligned}
2 a_{2}+2 a_{3}^{\prime}-a_{3}^{\prime \prime} & =0, \quad 2 a_{1}-a_{3}^{\prime}+2 a_{3}^{\prime \prime}=0, \\
a_{1}+a_{2} & =0, \quad a_{3}^{\prime}+a_{3}^{\prime \prime}=0,
\end{aligned}
$$

with a nonzero solution $a_{1}=1.5, a_{2}=-1.5, a_{3}^{\prime}=1, a_{3}^{\prime \prime}=-1$. Easy calculations 
show that the standard generator of $N_{3}\left(3,1^{2}\right)$ is

$$
f\left(x_{1}, x_{2}, x_{3}\right)=S_{3}\left(x_{1}, x_{2}, x_{3}\right) x_{1}^{2}(-2 \mathrm{id}+3(34)+3(35)),
$$

where id, (34), (35) $\in \operatorname{Sym}(5)$.

The direct product $\operatorname{Sym}(p) \times \operatorname{Sym}(q)$ is canonically embedded into $\operatorname{Sym}(p+q), \operatorname{Sym}(p)$ acting on $\{1, \ldots, p\}$ and $\operatorname{Sym}(q)$ on $\{p+1, \ldots, p+q\}$. Moreover, for a subgroup $H$ of the group $G$ and $M$ being an $H$-module, we denote by $M \uparrow G$ the $G$-module induced by $M$. The Littlewood-Richardson rule has the following interpretation in the language of $\operatorname{Sym}(n)$-representations.

Then

1.3.6. TheOREM. Let $\|\lambda\|=p,\|\mu\|=q$ and $N_{m}(\hat{\lambda}) \otimes_{K} N_{m}(\mu) \cong \sum c_{\lambda \mu}^{v} N_{m}(v)$.

$$
\left(M(\lambda) \otimes_{K} M(\mu)\right) \uparrow \operatorname{Sym}(p+q) \cong \sum c_{\lambda \mu}^{\nu} M(v),
$$

where $M(\lambda) \otimes_{K} M(v)$ has a structure of $\operatorname{Sym}(p) \times \operatorname{Sym}(q)$-module.

\subsection{Other products of modules}

In this section we shortly discuss some other products of modules which have applications to PI-algebras.

1.4.1. The Kronecker product. Let $\lambda$ and $\mu$ be partitions of $n$. Then the Kronecker (or inner) product $M(\lambda) \otimes_{K} M(\mu)$ of $M(\hat{\lambda})$ and $M(\mu)$ is a $\operatorname{Sym}(n)$-module with a diagonal action of $\operatorname{Sym}(n)$. This product plays an important role for computing the cocharacter sequence of the $k \times k$ matrix algebra (see e.g. [56]).

1.4.2. Symmetrized tensor powers. For a $\mathrm{GL}_{m}$-module $N$ we consider the symmetrized tensor power

$$
N \stackrel{\mathbf{s}}{\otimes k}=N \stackrel{\mathbf{s}}{\otimes} \ldots \stackrel{\mathbf{s}}{\otimes} N
$$

identifying the tensors $v_{1} \otimes \ldots \otimes v_{k}$ and $v_{\sigma(1)} \otimes \ldots \otimes v_{\sigma(k)}, \sigma \in \operatorname{Sym}(k), v_{i} \in N$. A result of Thrall [63] (see also [41], Exercise 5, p. 45) shows that

$$
N_{m}(2) \stackrel{s}{\otimes k} \cong \sum N_{m}\left(2 \lambda_{1}, \ldots, 2 \lambda_{m}\right) \text {. }
$$

The symmetrized tensor product is a very special case of the general notion of plethysm (see $[31,41]$ ).

\subsection{Representations of Lie superalgebras}

In this survey we apply the representation theory of $\operatorname{Sym}(n)$ and $\mathrm{GL}_{m}$ or equivalently, of the Lie algebra $\mathrm{gl}_{m}$. This theory works very well when the number of rows of all the Young diagrams appearing in the decomposition $P_{n}(U)=\sum k(\lambda) M(\lambda), n=1,2, \ldots$, is bounded. Generally, the number of rows increases with $n$ and the behaviour of $P_{n}(U)$ cannot be studied by the representations of a fixed $\mathrm{GL}_{m}$. Kemer (see $[32,34]$ ) has applied $\mathbf{Z}_{2}$-graded algebras for the investigation of associative PI-algebras. This has allowed him 
to obtain important results on polynomial identities. In particular, he has built the structure theory of $T$-ideals in $K\langle X\rangle$ in the spirit of commutative algebra. A combination of [53] and [32] gives the following theorem whose final version is due to Braun [8].

1.5.1. TheOREM (Razmyslov-Kemer-Braun [53, 32, 8]). The Jacobson radical of every finitely generated associative PI-algebra (i.e. with nontrivial identity from $K\langle X\rangle$ ) over an arbitrary field is nilpotent.

In practice, the application of $\mathbf{Z}_{2}$-graded algebras involves the representation theory of Lie superalgebras. The formalism of representations of linear Lie superalgebras has been developed by Berele and Regev [7] and they have obtained important quantitative results in the associative case.

\section{First applications}

\subsection{Relatively free algebras}

One of the main problems in this paper is the following: How to compute the cocharacter sequence of a $T$-ideal or, equivalently, to find the multiplicities $k(\lambda)$ of the irreducible $\mathrm{GL}_{m}$-submodules of $F_{m}(U)$ ? A trivial example is the free associative algebra $A_{m}$, when the $\operatorname{Sym}(n)$-modules $P A_{n} \subset A$ and $K \operatorname{Sym}(n)$ are isomorphic. Therefore, $A_{m}=\sum(\operatorname{dim} M(\lambda)) N_{m}(\lambda)$. For small $n$ it is possible to use the character table of $\operatorname{Sym}(n)$.

2.1.1. ExAMPLE. Let $P L_{4}$ be the set of all multilinear elements of degree 4 in the free Lie algebra $L$. It is well known (see e.g. [4]) that $P L_{4}$ has a basis $\left\{x_{4} x_{\sigma(1)} x_{\sigma(2)} x_{\sigma(3)} \mid \sigma \in \operatorname{Sym}(3)\right\}$. Let $\chi$ be the character of the $\operatorname{Sym}(4)$-module $P L_{4}$ and let $\chi=\sum k(\lambda) \chi_{\lambda}$. Easy calculations show that $\chi(\mathrm{id})=\operatorname{dim} P L_{4}=6, \chi(12)$ $=\chi(123)=\chi(1234)=0, \chi((12)(34))=-2$. The character table of Sym(4) is the following:

\begin{tabular}{rrrrrr}
\hline & id & $(12)$ & $(123)$ & $(1234)$ & $(12)(34)$ \\
\hline$(4)$ & 1 & 1 & 1 & 1 & 1 \\
$(3,1)$ & 3 & 1 & 0 & -1 & -1 \\
$\left(2^{2}\right)$ & 2 & 0 & -1 & 0 & 2 \\
$\left(2,1^{2}\right)$ & 3 & -1 & 0 & 1 & -1 \\
$\left(1^{4}\right)$ & 1 & -1 & 1 & -1 & 1 \\
\hline
\end{tabular}

Hence we obtain a linear system for $k(\lambda)$ :

$$
\begin{aligned}
\chi(\mathrm{id}) & =k(4)+3 k(3,1)+2 k\left(2^{2}\right)+3 k\left(2,1^{2}\right)+k\left(1^{4}\right)=6, \\
\chi(12) & =k(4)+k(3,1)-k\left(2,1^{2}\right)-k\left(1^{4}\right)=0, \\
\chi(123) & =k(4)-k\left(2^{2}\right)+k\left(1^{4}\right)=0, \\
\chi(1234) & =k(4)-k(3,1)+k\left(2,1^{2}\right)-k\left(1^{4}\right)=0, \\
\chi((12)(34)) & =k(4)-k(3,1)+2 k\left(2^{2}\right)-k\left(2,1^{2}\right)+k\left(1^{4}\right)=-2 .
\end{aligned}
$$


The only solution of the system is $k(4)=k\left(2^{2}\right)=k\left(1^{4}\right)=0, k(3,1)=k\left(2,1^{2}\right)$ $=1$; therefore $P L_{4}=M(3,1)+M\left(2,1^{2}\right)$. In practice, bearing in mind that we are interested in solutions in nonnegative integers, it suffices to consider only a part of the equations.

2.1.2. The same example. The dimension of $M(\lambda)$ equals the number of standard $\lambda$-tableaux (i.e. the semistandard tableaux with content $(1, \ldots, 1)$ ). The hook formula gives another expression for $\operatorname{dim} M(\lambda)$ :

$$
\operatorname{dim} M(\lambda)=n ! / \prod\left(\lambda_{i}+\lambda_{j}^{\prime}-i-j+1\right),
$$

where $\lambda_{j}^{\prime}$ is the length of the $j$ th column of [ $\left.\lambda\right]$. Applying one of these two expressions for $\operatorname{dim} M(\hat{\lambda})$, it is easy to show that $\operatorname{dim} M(3,1)=$ $\operatorname{dim} M\left(2,1^{2}\right)=3$ and hence $\operatorname{dim} P L_{4}=\operatorname{dim} M(3,1)+\operatorname{dim} M\left(2,1^{2}\right)$. Therefore, in virtue of Theorem 1.2.1 it suffices to obtain in the free Lie algebra nonzero standard generators for $N_{m}(3,1)$ and $N_{m}\left(2,1^{2}\right)$. But

$$
f_{(3,1)}=\left(x_{1} x_{2}-x_{2} x_{1}\right) x_{1} x_{1} \text { and } f_{\left(2,1^{2}\right)}=\sum(\operatorname{sign} \sigma)\left(x_{1} x_{\sigma(1)}\right)\left(x_{\sigma(2)} x_{\sigma(3)}\right)
$$

do not vanish in $L_{m}$ and this gives the desired decomposition.

This method has been used successfully to obtain similar decompositions for $P_{n}(U), n \geqslant 1$, for the varieties of Lie algebras $U_{1}=N_{2} A \cap A N_{2}$ determined by the identities $\left(x_{1} x_{2}\right)\left(x_{3} x_{4}\right)\left(x_{5} x_{6}\right)$ and $\left(x_{1} x_{2} x_{3}\right)\left(x_{4} x_{5} x_{6}\right)$ [10] and $\boldsymbol{U}_{2}=\left[\boldsymbol{A}^{2}, \boldsymbol{E}, \boldsymbol{E}\right]$ defined by $\left(x_{1} x_{2}\right)\left(x_{3} x_{4}\right) x_{5} x_{6}$ [43]. For other applications see $[5,22]$.

2.1.3. THEOREM [63]. Let $L_{m}^{(n)}$ be the homogeneous component of degree $n$ of the free algebra $L_{m}$. Then the following $\mathrm{GL}_{m}$-module isomorphism holds:

$$
K+A_{m} \cong \sum\left(\left(L_{m}^{(1)}\right)^{\stackrel{s}{\otimes} p_{1}} \otimes_{K} \ldots \otimes_{K}\left(L_{m}^{(r)}\right)^{\stackrel{s}{\otimes} p_{r}}\right)
$$

where the sum is over all symmetrized tensor powers with $p_{i} \geqslant 0$.

Proof. It is known that the free associative algebra $A_{m}$ coincides with the universal enveloping algebra of $L_{m}$. Let $\left\{g_{i j} \mid j=1, \ldots, d_{i}\right\}$ be a multihomogeneous basis of the vector space $L_{m}^{(i)}$. By the Poincaré-Birkhoff-Witt theorem, $K+A_{m}$ has a basis $\left\{\prod_{i}\left(\prod_{j} g_{i j}^{a_{i j}}\right) \mid a_{i j} \geqslant 0\right\}$. Hence the Hilbert series of $K+A_{m}$ equals the Hilbert series of

$$
\sum\left(\left(L_{m}^{(1)}\right)^{\stackrel{s}{\otimes} p_{1}} \otimes_{K} \ldots \otimes_{K}\left(L_{m}^{(r)}\right)^{\stackrel{s}{\otimes} p_{r}}\right) .
$$

Since the Hilbert series determines uniquely the $\mathrm{GL}_{m}$-module, the desired isomorphism holds.

Since the $\mathrm{GL}_{m}$-module structure of $A_{m}^{(n)}$ is known, Theorem 2.1.3 allows one to calculate the structure of $L_{m}^{(n)}$. This has been done for $n \leqslant 10$ in [63]. For example, $\quad L_{m}^{(1)}=N_{m}(1), L_{m}^{(2)}=N_{m}\left(1^{2}\right), L_{m}^{(3)}=N_{m}(2,1) \quad$ and $\quad L_{m}^{(4)}=N_{m}(3,1)$ 
$+N_{m}\left(2,1^{2}\right)$. Therefore

$$
\begin{aligned}
A_{m}^{(5)}= & \left(L_{m}^{(1)}\right)^{\stackrel{\mathrm{s}}{\otimes} 5}+\left(L_{m}^{(1)}\right)^{\stackrel{\mathrm{s}}{\otimes}} \otimes_{K} L_{m}^{(2)}+\left(L_{m}^{(1)}\right)^{\stackrel{\mathrm{s}}{\otimes}} \otimes_{K} L_{m}^{(3)} \\
& +L_{m}^{(1)} \otimes_{K}\left(L_{m}^{(4)}+\left(L_{m}^{(2)}\right)^{\mathrm{s}} 2\right)+L_{m}^{(2)} \otimes_{K} L_{m}^{(3)}+L_{m}^{(5)} \\
= & N_{m}(5)+N_{m}(3) \otimes_{K} N_{m}\left(1^{2}\right)+N_{m}(2) \otimes_{K} N_{m}(2,1) \\
& +N_{m}(1) \otimes_{K}\left(\left(N_{m}(3,1)+N_{m}\left(2,1^{2}\right)\right)+\left(N_{m}\left(2^{2}\right)+N_{m}\left(1^{4}\right)\right)\right) \\
& +N_{m}\left(1^{2}\right) \otimes_{K} N_{m}(2,1)+L_{m}^{(5)}
\end{aligned}
$$

and bearing in mind that $A_{m}^{(5)}=N_{m}(5)+4 N_{m}(4,1)+5 N_{m}(3,2)+6 N_{m}\left(3,1^{2}\right)$ $+5 N_{m}\left(2^{2}, 1\right)+4 N_{m}\left(2,1^{3}\right)+N_{m}\left(1^{5}\right)$ we establish

$$
L_{m}^{(5)}=N_{m}(4,1)+N_{m}(3,2)+N_{m}\left(3,1^{2}\right)+N_{m}\left(2^{2}, 1\right)+N_{m}\left(2,1^{3}\right) .
$$

The standard generators of $N_{m}(\lambda) \subset L_{m}^{(n)}$ for $n \leqslant 6$ have been obtained in [10, 12]. Another approach to the free Lie algebra is given in [35] (see also [4]).

2.1.4. Example. Let $C_{m}$ be the free commutative algebra of rank $m$, i.e. the relatively free algebra $F_{m}(C)$ of the variety defined by the identity $x_{1} x_{2}-x_{2} x_{1}$. It follows from [58] that if $C_{m}^{(i)}$ has a basis $\left\{u_{i j} \mid j=1, \ldots, d_{i}\right\}, i<n$, then $C_{m}^{(n)}$ has a basis

$$
\begin{gathered}
\left\{u_{i j} u_{n-i, k} \mid j=1, \ldots, d_{i}, k=1, \ldots, d_{n-i}, i=1, \ldots,[n / 2],\right. \\
\text { and if } i=n-i \text { then } j \leqslant k\} .
\end{gathered}
$$

As in the proof of Theorem 2.1.3,

$$
C_{m}^{(n)} \cong \sum_{i<n-i} C_{m}^{(i)} \otimes_{K} C_{m}^{(n-i)}+\varepsilon C_{m}^{(n / 2)} \stackrel{s}{\otimes}_{K} C_{m}^{(n / 2)}
$$

where $\varepsilon=1$ for $n$ even and $\varepsilon=0$ for $n$ odd. In particular, $C_{m}^{(1)}=N_{m}(1)$,

$$
\begin{aligned}
C_{m}^{(2)} \cong N_{m}(1) \otimes_{K} N_{m}(1) \cong N_{m}(2), \quad C_{m}^{(3)} \cong C_{m}^{(1)} \otimes_{K} C_{m}^{(2)} \cong N_{m}(3)+N_{m}(2,1), \\
C_{m}^{(4)} \cong C_{m}^{(1)} \otimes_{K} C_{m}^{(3)}+C_{m}^{(2)} \otimes_{K} C_{m}^{(2)} \cong 2 N_{m}(4)+2 N_{m}(3,1)+2 N_{m}\left(2^{2}\right)+N_{m}\left(2,1^{2}\right), \\
C_{m}^{(5)} \cong C_{m}^{(1)} \otimes_{K} C_{m}^{(4)}+C_{m}^{(2)} \otimes_{K} C_{m}^{(3)} \\
\cong 3 N_{m}(5)+6 N_{m}(4,1)+6 N_{m}(3,2)+4 N_{m}\left(3,1^{2}\right)+4 N_{m}\left(2^{2}, 1\right)+N_{m}\left(2,1^{3}\right)
\end{aligned}
$$

The standard generators of the submodules can be obtained by Rule 1.3.4 or its modification for symmetrized tensor powers. Another method for computing $C_{m}^{(5)}$ is applied in [46]. Similar formulas can be established for the free anticommutative algebra when in (3) the symmetrized tensor square has to be replaced by the antisymmetrized tensor square. 
2.1.5. Example [23]. Let $S_{2}$ be the variety of all solvable Jordan algebras of class 2 (i.e. $S_{2}$ is defined by $\left(x_{1} x_{2}\right)\left(x_{3} x_{4}\right)$ ). Then the vector space $F\left(S_{2}\right)$ has a basis

$$
\left\{x_{i_{1}} x_{i_{2}} \ldots x_{i_{n}} \mid i_{1} \geqslant i_{2}<i_{4}<i_{6}<\ldots, i_{3}<i_{5}<\ldots\right\} \text {. }
$$

The Hilbert series of the subspaces of $A_{m}^{(p)}$ spanned by $\left\{x_{j_{1}} x_{j_{2}} \ldots x_{j_{p}} \mid j_{1} \geqslant j_{2}<j_{3}\right.$ $\left.<\ldots<j_{p}\right\}$ and $\left\{x_{k_{1}} \ldots x_{k_{p}} \mid k_{1}<\ldots<k_{p}\right\}$ coincide with the Hilbert series of $N_{m}\left(2,1^{p-2}\right)$ and $N_{m}\left(1^{p}\right)$, respectively. Hence $F_{m}^{(2 k+1)}\left(S_{2}\right) \cong N_{m}\left(2,1^{k-1}\right) \otimes_{K} N_{m}\left(1^{k}\right)$ and $F_{m}^{(2 k+2)}\left(S_{2}\right) \cong N_{m}\left(2,1^{k}\right) \otimes_{K} N_{m}\left(1^{k}\right), k \geqslant 1$, and the Littlewood-Richardson rule allows one to calculate the cocharacter sequence of the variety $S_{2}$.

\subsection{Unitary algebras}

In this section we consider varieties $U$ of unitary algebras only. We denote by $F^{\#}=K\{X\}^{\#}$ the absolutely free unitary algebra and by $F^{\#}(\boldsymbol{U})$ the free algebra of the variety $U$. In the associative case it is known that every variety $\boldsymbol{U}$ is determined by its proper (or commutator) multilinear identities [60]. A similar result holds for arbitrary algebras. For $f\left(x_{1}, \ldots, x_{m}\right) \in F^{\#}$, let $\partial f / \partial x_{i}$ be the formal derivative in $x_{i}$. For a multihomogeneous polynomial, $\partial f / \partial x_{i}$ equals $f\left(x_{1}|\ldots| x_{i}, 1|\ldots| x_{m}\right)$. We write

$$
B_{m}=\left\{f \in F_{m}^{\#} \mid \partial f / \partial x_{i}=0, i=1, \ldots, m\right\} \quad \text { and } \quad \Gamma_{n}=P_{n} \cap B_{n}
$$

for the subspace of $F_{m}^{\#}$ vanishing under the formal derivations and the space of proper multilinear polynomials of degree $n$, respectively. It is easy to see that an analogue of Theorem 1.2.1 holds and $\Gamma_{n}(U)$ and $B_{m}^{(n)}(U)$ have the same module structure.

2.2.1. THEOREM $[13,14,16]$. (i) Every polynomial from $F_{m}(U)$ can be uniquely written in the form $\sum b_{i}\left(x_{1}, \ldots, x_{m}\right) x_{i_{1}} \ldots x_{i_{n}}$, where $b_{i} \in B_{m}(U)$ and $i_{1} \leqslant \ldots \leqslant i_{n}$.

(ii) Any variety $U$ can be defined $b_{y}$ its identities from $\Gamma_{n}, n=2,3, \ldots$

(iii) $H\left(\boldsymbol{U}, t_{1}, \ldots, t_{m}\right)=H\left(B_{m}(U), t_{1}, \ldots, t_{m}\right) / \prod_{i=1}^{m}\left(1-t_{i}\right), H_{m}(U, t)=$ $=H\left(B_{m}(U), t\right) /(1-t)^{m}$.

(iv) The following $\mathrm{GL}_{m}$-module isomorphism is valid:

$$
F_{m}(U) \cong B_{m}(U) \otimes_{K} K\left[x_{1}, \ldots, x_{m}\right]^{\#},
$$

where $K\left[x_{1}, \ldots, x_{m}\right]^{\#}$ is the ordinary algebra of polynomials in commuting variables.

Since $K\left[x_{1}, \ldots, x_{m}\right]^{\#} \cong \sum_{n \geq 0} N_{m}(n)$, Theorem 2.2.1 and Corollary 1.3.3 reduce the problem of decomposition of $F_{m}^{\#}(U)$ to a similar (but simpler) problem of decomposition of $B_{m}(U)$. In particular:

2.2.2. Corollari [13]. Let

$$
F_{m}^{\#}(\boldsymbol{U})=\sum k\left(\hat{\lambda}_{1}, \ldots, \lambda_{m}\right) N_{m}(\hat{\lambda}), \quad B_{m}(\boldsymbol{U})=\sum k_{1}\left(\mu_{1}, \ldots, \mu_{m}\right) N_{m}(\mu) .
$$


Then $k\left(\lambda_{1}, \ldots, \lambda_{m}\right)=\sum k_{1}\left(\mu_{1}, \ldots, \mu_{m}\right)$, where the summation is over all partitions $\left(\mu_{1}, \ldots, \mu_{m}\right)$ such that $\lambda_{1} \geqslant \mu_{1} \geqslant \lambda_{2} \geqslant \mu_{2} \geqslant \ldots \geqslant \lambda_{m} \geqslant \mu_{m}$.

Especially for the free associative algebra $A_{m}^{\#}$ there exists a good basis of the space $B A_{m}$ of proper polynomials in $A_{m}^{\#}$.

2.2.3. Proposition [59]. The vector space $B A_{m} \subset A_{m}^{\#}$ has the following basis: $\left[x_{i_{1}}, \ldots, x_{i_{p}}\right] \ldots\left[x_{j_{1}}, \ldots, x_{j_{q}}\right]$, where $i_{1}>i_{2} \leqslant \ldots \leqslant i_{p}, \ldots, j_{1}>j_{2}$ $\leqslant \ldots \leqslant j_{q}$, and $[u, v]=u v-v u,\left[u_{1}, \ldots, u_{p}\right]=\left[\left[u_{1}, \ldots, u_{p-1}\right], u_{p}\right]$.

Additional arguments give an expression for the $\mathrm{GL}_{m}$-module $B A_{m}$ :

2.2.4. ThEOREM [20]. The following $\mathrm{GL}_{m}$-module isomorphism holds:

$$
B A_{m} \cong \sum N_{m}\left(p_{1}-1,1\right) \otimes_{K} \ldots \otimes_{K} N_{m}\left(p_{r}-1,1\right),
$$

where the summation runs over all integers $p_{i} \geqslant 2, r=0,1, \ldots$

In particular, modulo the $T$-ideal of $A_{m}$ generated by $\left[x_{1}, x_{2}\right]\left[x_{3}, x_{4}\right]$, the commutators $\left[x_{i_{1}}, \ldots, x_{i_{p}}\right], i_{1}>i_{2} \leqslant \ldots \leqslant i_{p}$, span the module $N_{m}(p-1,1)$. Hence Rule 1.3.4, Proposition 2.2.3 and Theorem 2.2.4 allow one to obtain the standard generators of the irreducible submodules of $B A_{m}^{(n)}$. For $n \leqslant 6$ this has been done in [12], for $n=7$ in [49] and for $n=8$ partially in [20].

\section{Simulation of $2 \times 2$ matrices}

\subsection{Algebras with good bases}

The three-dimensional real vector space $\mathbf{R}^{3}$ with the usual scalar and vector products enjoys the following properties:

(i) For every basis $f_{1}, f_{2}, f_{3}$ of $\mathbf{R}^{3}$ the standard process of orthogonalization gives an orthogonal basis $e_{1}, e_{2}, e_{3}$.

(ii) Let $g\left(x_{1}, x_{2}, x_{3}\right)=\left(x_{i_{1}} \times \ldots\right) \times\left(\ldots \times x_{i_{n}}\right)$ be a monomial (with respect to the vector product) of degree $d_{i}$ in $x_{i}$. Then $g\left(e_{1}, e_{2}, e_{3}\right)=\varepsilon e_{1}^{\delta_{1}} \times e_{2}^{\delta_{2}} \times e_{3}^{\delta_{3}}$, where $\varepsilon=0, \pm 1, \delta_{i}=0,1, \delta_{i} \equiv d_{i}(\bmod 2)\left(\right.$ and $\left.e_{1}^{0} \times e_{2}^{0} \times e_{3}^{0}=0\right)$.

It turns out that these simple properties of $\mathbf{R}^{3}$ play an important role in the investigation of the polynomial identities of $2 \times 2$ matrices [10]. Here we give a generalization which works successfully in several different cases.

In virtue of Proposition 1.1.2, we assume that the base field is algebraically closed. We make use of the Zariski topology [30, pp. 36-37]. Let $u_{1}, \ldots, u_{k}$ be a fixed basis of a vector space $W, K[y]^{\#}=K\left[y_{i j} \mid i=1, \ldots, k, j=1, \ldots, m\right]^{\#}$ the polynomial algebra over $K$ and $Q$ a subset of $K[y]^{\#}$. The set of all $m$-tuples of vectors $\left(v_{1}, \ldots, v_{m}\right) \in W^{m}, v_{j}=\sum_{i=1}^{k} \xi_{i j} u_{i}, \xi_{i j} \in K$, such that $g\left(\xi_{i j}\right)=0$ for every $g\left(y_{i j}\right) \in Q$ is closed in the Zariski topology. Any nonempty open subset is dense in this topology. Hence a polynomial function which vanishes on an open subset vanishes everywhere. In particular, if $R$ is a finite-dimensional algebra and 
$f\left(x_{1}, \ldots, x_{m}\right) \in K\{X\}$ and $f\left(r_{1}, \ldots, r_{m}\right)=0$ on a nonempty open subset of $R^{m}$, then $f\left(x_{1}, \ldots, x_{m}\right)$ is a polynomial identity for $R$.

3.1.1. Definition. Let $R$ be a finite-dimensional algebra. We call the basis $r_{1}, \ldots, r_{p}$ of $R$ good if there èxists a $p \times p$ upper-triangular matrix $U=\left(\alpha_{i j}\right)$, $\alpha_{i i}=1$, with the following property:

for any two monomials $g_{1}\left(x_{1}, \ldots, x_{p}\right), g_{2}\left(x_{1}, \ldots, x_{p}\right) \in K\{X\}$ of degree $d_{j}$ in $x_{j}$ there exist $\eta_{1}, \eta_{2} \in K,\left(\eta_{1}, \eta_{2}\right) \neq(0,0), \eta_{1}, \eta_{2}$ depending on $g_{1}$ and $g_{2}$ only, such that

$$
\eta_{1} g_{1}\left(s_{1}, \ldots, s_{p}\right)=\eta_{2} g_{2}\left(s_{1}, \ldots, s_{p}\right) \text {, where } s_{j}=\sum_{i=1}^{j} \alpha_{i j} r_{i}, j=1, \ldots, p .
$$

3.1.2. TheOREM. Let $R$ be a finite-dimensional algebra, $\operatorname{dim} R=p$ and let the set of all good bases in $R$ be dense in the Zariski topology in $R^{p}$. Then for the variety $\operatorname{var} R$ generated by $R$ the $\mathrm{GL}_{m}$-module $F_{m}(\operatorname{var} R)$ is a submodule of $\sum N_{m}\left(\lambda_{1}, \ldots, \lambda_{p}\right)$, i.e. the multiplicities of the irreducible submodules $N_{m}(\lambda)$ of $F_{m}(\operatorname{var} R)$ equal 0 or 1 and are zero if $\lambda_{p+1} \neq 0$.

Proof. Let $F_{m}(\operatorname{var} R)=\sum k(\lambda) N_{m}(\lambda)$ and let $\lambda=\left(\lambda_{1}, \ldots, \lambda_{q}\right)$ be a partition, $\lambda_{q} \neq 0$. First, assume that $q>p$ and that $f_{\lambda}\left(x_{1}, \ldots, x_{q}\right) \in F_{m}$ is a standard generator of $N_{m}(\lambda)$. Since arbitrary $u_{1}, \ldots, u_{q} \in R$ are linearly dependent and there is a skew symmetry in the $q$ variables of $f_{\lambda}$ (see (1) and $\left(1^{\prime}\right)$ ), $f_{\lambda}\left(u_{1}, \ldots, u_{q}\right)=0$ and $f_{\lambda}$ is a polynomial identity for $R$, i.e. $k(\lambda)=0$ if $\lambda_{p+1} \neq 0$. Now, let $q \leqslant p$ and let $f_{\lambda}^{\prime}, f_{\lambda}^{\prime \prime} \in F_{m}$ be standard generators of two isomorphic copies of $N_{m}(\lambda)$. Let $r_{1}, \ldots, r_{p}$ be a good basis and $s_{1}, \ldots, s_{p}$ be the related vectors from Definition 3.1.1. Therefore, for suitable $\beta_{i j} \in K, r_{1}=s_{1}, r_{2}$ $=s_{2}+\beta_{12} s_{1}, \ldots, r_{p}=s_{p}+\beta_{1 p} s_{1}+\ldots+\beta_{p-1, p} s_{p-1}$. Bearing in mind the skew symmetry in (1) and $\left(1^{\prime}\right)$ we obtain $f^{\prime}\left(r_{1}, \ldots, r_{q}\right)=f^{\prime}\left(s_{1}, \ldots, s_{q}\right)$, $f^{\prime \prime}\left(r_{1}, \ldots, r_{q}\right)=f^{\prime \prime}\left(s_{1}, \ldots, s_{q}\right)$. Since $f_{\lambda}^{\prime}$ and $f_{\lambda}^{\prime \prime}$ are multihomogeneous of degree $\lambda_{j}$ in $x_{j}$. Definition 3.1 .1 gives that either $f_{\lambda}^{\prime}\left(s_{1}, \ldots, s_{q}\right)=f_{\lambda}^{\prime \prime}\left(s_{1}, \ldots, s_{q}\right)=0$ or $f_{\lambda}^{\prime}\left(s_{1}, \ldots, s_{q}\right) \neq 0$ and there exists $v \in K, v \neq 0$, such that $f_{\lambda}^{\prime \prime}\left(s_{1}, \ldots, s_{q}\right)$ $-v f_{\lambda}^{\prime}\left(s_{1}, \ldots, s_{q}\right)=0$. Hence $f_{\lambda}\left(x_{1}, \ldots, x_{q}\right)=f_{\lambda}^{\prime \prime}\left(x_{1}, \ldots, x_{q}\right)-v f_{\lambda}^{\prime}\left(x_{1}, \ldots, x_{q}\right)$ is a standard generator of $N_{m}(\lambda)$ (which does not depend on the choice of the basis $\left.r_{1}, \ldots, r_{p}\right)$ and $f_{\lambda}\left(r_{1}, \ldots, r_{q}\right)=0$. Since the set of good bases is dense in $R^{p}$ this yields that $f_{\lambda}\left(x_{1}, \ldots, x_{q}\right)$ is a polynomial identity for $R$. Hence the two isomorphic copies of $N_{m}(\lambda)$ are "glued together" in $F_{m}(\operatorname{var} R)$ and $k(\lambda) \leqslant 1$.

The subvarieties of a variety $\boldsymbol{U}$ form a lattice with respect to the intersection and union. It is distributive if and only if $P_{n}(U), n=1,2, \ldots$, are sums of nonisomorphic irreducible submodules.

3.1.3. COROLlaRY. Under the conditions of Theorem 3.1.2, the lattice of subvarieties of $\operatorname{var} R$ is distributive.

A modification of Theorem 3.1.2 works for varieties of unitary algebras. For 
a unitary algebra $R$ we define

$$
Z(R)=\{z \in R \mid z r=r z,(z r) s=r(z s)=z(r s) \text { for all } r, s \in R\}
$$

and fix a decomposition $R=Z(R) \oplus S$ into a direct sum of vector spaces.

3.1.4. Definition. Let $\operatorname{dim} R / Z(R)<\infty$ and $R=Z(R) \oplus S$. The basis $r_{1}, \ldots, r_{n}$ of $S$ is good if it has the property described in Definition 3.1.1.

3.1.5. Theorem. Let $R=Z(R) \oplus S, \operatorname{dim} S=p$ and let the set of all good bases of $S$ be dense in $S^{p}$. Then $B_{m}(\operatorname{var} R)$ is a submodule of $\sum N_{m}\left(\lambda_{1}, \ldots, \lambda_{p}\right)$.

Proof. Let $f\left(x_{1}, \ldots, x_{m}\right) \in B_{m}$ and $u_{j}=z_{j}+v_{j} \in R, z_{j} \in Z(R), v_{j} \in S$, $j=1, \ldots, m$. Since we have $\partial f / \hat{\imath} x_{j}=0$ and $z_{j} \in Z(R)$, it follows that $f\left(z_{1}+v_{1}, \ldots, z_{m}+v_{m}\right)=f\left(v_{1}, \ldots, v_{m}\right)$, i.e. $f\left(x_{1}, \ldots, x_{m}\right)$ is an identity for $R$ if and only if $f\left(v_{1}, \ldots, v_{m}\right)=0$ for all $v_{j} \in S$. The proof is completed by repeating verhatim the arguments of the proof of Theorem 3.1.2.

3.1.6. Corollary. In Theorem 3.1.5, the lattice of sublarieties of $\operatorname{var} R$ is distributive.

\subsection{The Grassmann algebra}

Let $V_{p}$ be a $p$-dimensional vector space with a basis $e_{1}, \ldots, e_{p}$. The Grassmann (or exterior) algebra $E_{p}=E\left(V_{p}\right)$ of $V_{p}$ has a basis $e_{i_{1}} \ldots e_{i_{q}}, 1 \leqslant i_{1}<\ldots<i_{q} \leqslant p, q \geqslant 0$, and the multiplication is defined by the associative law and $e_{i} e_{j}=-e_{j} e_{i}$. The polynomial identities of $E=E_{\infty}$ have been described in [37] (see also [2]). Here we give an alternative exposition.

3.2.1. Theorem. (i) Let $\Gamma_{n}(\operatorname{var} E)$ be the set of proper multilinear polynomials in $F^{\#}(\operatorname{var} E)$. Then $\Gamma_{n}(\operatorname{var} E)=M\left(1^{n}\right)$ for $n$ even and $\Gamma_{n}(\operatorname{var} E)=0$ for $n$ odd.

(ii) $P_{n}(\operatorname{var} E)=\sum_{q=1}^{n} M\left(q, 1^{n-q}\right)$.

Proof. (i) The algebra $E$ is $\mathrm{Z}_{2}$-graded, $E=E^{0} \oplus E^{1}, E^{0}$ and $E^{1}$ being spanned by the monomials $e_{i_{1}} \ldots e_{i_{q}}$ of even and odd degree, respectively. Since we have $Z(E)=E^{0}$ and $S=E^{1} \ni s=\alpha_{1} e_{j_{1}}+\ldots+\alpha_{p} e_{j_{p}}, \alpha_{i} \in E^{0}$, it follows that $r_{\sigma(1)} \ldots r_{\sigma(n)}=(\operatorname{sign} \sigma) r_{1} \ldots r_{n}$ for arbitrary elements $r_{1}, \ldots, r_{n} \in S$. In particular, if $r_{i}=r_{j}, i \neq j$, then $r_{1} \ldots r_{n}=0$. For studying the polynomial identities of degree $n$, without loss of generality we investigate $E_{p}$ instead of $E, p$ being sufficiently large, and replace the free algebra $K\{X\}^{\#}$ by the free associative algebra $A^{\#}=K\langle X\rangle^{\#}$. In virtue of Theorem 3.1.5, $B_{m}\left(\operatorname{var} E_{p}\right)$ (and hence $B_{m}(\operatorname{var} E)$ ) is a submodule of $\sum N_{m}\left(\lambda_{1}, \ldots, \lambda_{m}\right)$. Let $\lambda=\left(\lambda_{1}, \ldots, \lambda_{m}\right)$ with $\lambda_{1}>1$. Then the standard generator $f_{\lambda}\left(x_{1}, \ldots, x_{m}\right)$ is of degree $\geqslant 2$ in $x_{1}$, hence it vanishes on $E$ and therefore $B_{m}(\operatorname{var} E) \subset \sum N_{m}\left(1^{n}\right)$. For $n$ even, the standard polynomial $S_{n}\left(x_{1}, \ldots, x_{n}\right) \in A_{m}^{\#}$ is in the space $B A_{m}$ of proper elements and $S_{n}\left(e_{1}, \ldots, e_{n}\right) \neq 0$. For $n$ odd, the only submodule $N_{m}\left(1^{n}\right)$ of $A_{m}^{\#}$ is generated by $S_{n}\left(x_{1}, \ldots, x_{n}\right)$ which does not belong to $B A_{m}$. Hence $B_{m}(\operatorname{var} E)=\sum N_{m}\left(1^{2 k}\right)$. 
The expression for $\Gamma_{n}(\operatorname{var} E)$ is obtained immediately, because $\Gamma_{n}(\operatorname{var} E)$ and $B_{m}^{(n)}(\operatorname{var} E)$ have the same module structure.

(ii) The assertion follows immediately from (i) and Corollary 2.2.2.

\subsection{The Lie algebra $\mathrm{sl}_{2}$}

Let $\mathrm{sl}_{2}$ be the Lie algebra of all traceless $2 \times 2$ matrices with multiplication $[u, v]=u v-v u$. Over an algebraically closed field, $\mathrm{sl}_{2}$ is isomorphic to the three-dimensional vector space $K^{3}$ with the usual vector product

$$
\left(x_{1}, x_{2}, x_{3}\right) \times\left(y_{1}, y_{2}, y_{3}\right)=\left(x_{2} y_{3}-x_{3} y_{2}, x_{3} y_{1}-x_{1} y_{3}, x_{1} y_{2}-x_{2} y_{1}\right) .
$$

The Killing form of $K^{3}$ is proportional to the scalar product $\left\langle\left(x_{1}, x_{2}, x_{3}\right),\left(y_{1}, y_{2}, y_{3}\right)\right\rangle=x_{1} y_{1}+x_{2} y_{2}+x_{3} y_{3}$.

3.3.1. TheOREM [10]. $F_{m}\left(\operatorname{varsl}_{2}\right)=N_{m}(1)+\sum N_{m}\left(\lambda_{1}, \lambda_{2}, \lambda_{3}\right)$, where the summation runs over all $\left(\lambda_{1}, \lambda_{2}, \lambda_{3}\right)$ such that $\lambda_{2}>0$ and $\lambda_{1} \not \equiv \lambda_{2}$ or $\lambda_{2} \not \equiv \lambda_{3}(\bmod 2)$.

Proof. We identify the Lie algebras $\mathrm{sl}_{2}$ and $K^{3}$. The basis $r_{1}, r_{2}, r_{3}$ of $K^{3}$ will be good if it can be transformed to an orthogonal basis $s_{1}, s_{2}, s_{3}$ (see Definition 3.1.1 and the very beginning of Section 3.1). Additionally, if $\lambda_{1} \equiv \lambda_{2} \equiv \lambda_{3} \equiv \varepsilon(\bmod 2), \varepsilon=0, \quad 1$, then $s_{1}^{\varepsilon} \times s_{2}^{\varepsilon} \times s_{3}^{\varepsilon}=0$ and therefore $f_{\lambda}\left(s_{1}, s_{2}, s_{3}\right)=0$. In virtue of Theorem 3.1.2, $F_{m}\left(\operatorname{varsl}_{2}\right) \subset \sum N_{m}\left(\lambda_{1}, \lambda_{2}, \lambda_{3}\right)$, where $\lambda_{1} \not \equiv \lambda_{2}$ or $\lambda_{2} \not \equiv \lambda_{3}(\bmod 2)$. Since the module $N_{m}\left(\lambda_{1}\right), \lambda_{1}>1$, does not appear in the decomposition of the free Lie algebra $L_{m}$ the proof will be completed if we construct standard generators $f_{\lambda}$ for all $N_{m}\left(\lambda_{1}, \lambda_{2}, \lambda_{3}\right)$ with $\lambda_{1} \not \equiv \lambda_{2}$ or $\lambda_{2} \not \equiv \lambda_{3}(\bmod 2)$ and $\lambda_{2}>0$, such that the $f_{\lambda}$ are not polynomial identities for $\mathrm{sl}_{2}$. We refer to [10] for the explicit construction of $f_{\lambda}$.

Actually we have proved that the standard generator $f_{\lambda}\left(x_{1}, x_{2}, x_{3}\right)$, $\lambda=\left(\lambda_{1}, \lambda_{2}, \lambda_{3}\right)$, is a polynomial identity for $\operatorname{sl}_{2}$ if and only if $f_{\lambda}\left(a_{1}, a_{2}, a_{3}\right)=0$ for a basis $a_{1}, a_{2}, a_{3}$ of $\mathrm{sl}_{2}$ such that the matrix of the Killing form is diagonal with respect to $a_{1}, a_{2}, a_{3}$. For example, the following is such a basis:

$$
a_{1}=-\left(e_{11}-e_{22}\right) \sqrt{-1} / 2, \quad a_{2}=\left(e_{12}+e_{21}\right) \sqrt{-1} / 2, \quad a_{3}=\left(e_{12}-e_{21}\right) / 2
$$

with multiplication $a_{1} a_{2}=-a_{2} a_{1}=a_{3} / 2, a_{2} a_{3}=-a_{3} a_{2}=a_{1} / 2, a_{3} a_{1}=-a_{1} a_{3}$ $=a_{2} / 2, a_{1}^{2}=a_{2}^{2}=a_{3}^{2}=-1 / 4$, hence $\left[a_{1}, a_{2}\right]=a_{3},\left[a_{2}, a_{3}\right]=a_{1},\left[a_{3}, a_{1}\right]$ $=a_{2}$. Therefore we obtain the following consequence.

3.3.2. CoRollaRY. The standard generator $f_{\lambda}\left(x_{1}, x_{2}, x_{3}\right), \lambda=\left(\lambda_{1}, \lambda_{2}, \lambda_{3}\right)$, is a polynomial identity for $\mathrm{sl}_{2}$ if and only if $f_{\lambda}\left(a_{1}, a_{2}, a_{3}\right)=0$, where $a_{1}, a_{2}, a_{3}$ are defined in (4).

Razmyslov [52] has discovered a basis for the $T$-ideal of $\mathrm{sl}_{2}$ (i.e. a system of generators of the $T$-ideal) consisting of three identities of degree 5. Later, Filippov [24] has reduced this basis to one identity. Comparing the decom- 
positions

$$
\begin{gathered}
P_{5}\left(\operatorname{varsl}_{2}\right)=M(4,1)+M(3,2)+M\left(2^{2}, 1\right) \quad(\text { see Theorem 3.3.1), and } \\
P L_{5}=M(4,1)+M(3,2)+M\left(3,1^{2}\right)+M\left(2^{2}, 1\right)+M\left(2,1^{3}\right)
\end{gathered}
$$

(the multilinear Lie elements of degree 5-this is a consequence of Theorem 2.1.3) we deduce that the Sym(5)-submodule of $P L_{5}$ of multilinear identities of degree 5 for $\mathrm{sl}_{2}$ is isomorphic to $M\left(3,1^{2}\right)+M\left(2,1^{3}\right)$. This allows Razmyslov's result to be restated in the following way.

3.3.3. THEOREM [52]. The elements from the free Lie algebra $L$

$$
\begin{gathered}
f_{\left(3,1^{2}\right)}=\sum(\operatorname{sign} \sigma)\left(x_{\sigma(1)} x_{\sigma(2)}\right)\left(x_{\sigma(3)} x_{1}\right) x_{1}, \\
f_{\left(2,1^{3}\right)}=\sum(\operatorname{sign} \sigma) x_{1} x_{\sigma(1)} x_{\sigma(2)} x_{\sigma(3)} x_{\sigma(4)}
\end{gathered}
$$

form a basis for the polynomial identities of $\mathrm{sl}_{2}$. (In [52] these polynomials are replaced by the equivalent ones, $\left(x_{1} x_{2}\right)\left(x_{1} x_{3}\right) x_{1}$ and $\sum(\operatorname{sign} \sigma) x_{5} x_{\sigma(1)} \ldots x_{\sigma(4)}$.)

It turns out that almost all identities for $\mathrm{sl}_{2}$ follow from $\left(x_{1} x_{2}\right)\left(x_{1} x_{3}\right) x_{1}$ and the Lie standard identity gives only the details in lower degrees.

3.3.4. ThEOREM [15]. Let $U \triangleleft L$ be the T-ideal of all polynomial identities for $\mathrm{sl}_{2}$ and let $V$ be the T-ideal of $L$ generated by $\left(x_{1} x_{2}\right)\left(x_{1} x_{3}\right) x_{1}$. Then all homogeneous elements of $U$ of degree $\geqslant 7$ are contained in $V$ and

$$
\left(U \cap L_{m}\right) /\left(V \cap L_{m}\right)=N_{m}\left(2,1^{3}\right)+N_{m}\left(2^{2}, 1^{2}\right) .
$$

The proof is based on a result by Nikolaev [45] which asserts that the polynomial identities in three variables for $\mathrm{sl}_{2}$ follow from $\left(x_{1} x_{2}\right)\left(x_{1} x_{3}\right) x_{1}$, and on the decomposition of $L_{m}^{(6)}$ and $L_{m}^{(7)}$ into a sum of irreducible $\mathrm{GL}_{m}$-modules.

\subsection{The $2 \times 2$ matrix algebra}

The centre of the $2 \times 2$ matrix algebra $M_{2}(K)$ consists of scalar matrices only and, as vector spaces, $M_{2}(K)=K \oplus \mathrm{sl}_{2}$. Again the basis $r_{1}, r_{2}, r_{3}$ of $\mathrm{sl}_{2}$ will be good if it can be transformed to a basis $s_{1}, s_{2}, s_{3}$ corresponding to a diagonal matrix of the Killing form of $\mathrm{sl}_{2}$. The proof of the following result makes use of Theorem 3.1.5 and is similar to the proof of Theorem 3.3.1.

3.4.1. Theorem [10]. $B_{m}\left(\operatorname{var} M_{2}(K)\right)=K+\sum N_{m}\left(\lambda_{1}, \lambda_{2}, \lambda_{3}\right)$, where the summation is over all $\lambda=\left(\lambda_{1}, \lambda_{2}, \lambda_{3}\right) \neq\left(1^{3}\right)$ with $\lambda_{2}>0$.

As a consequence of Corollary 2.2.2 and Theorem 3.4.1 we obtain immediately

3.4.2. THEOREM $[13,26,51]$. Let $F_{m}\left(\operatorname{var} M_{2}(K)\right)=K+\sum k(\lambda) N_{m}(\lambda)$, $\lambda=\left(\lambda_{1}, \ldots, \lambda_{r}\right)$. Then: (i) $k(\lambda)=0$ if $\lambda_{5} \neq 0$; (ii) $k\left(\lambda_{1}\right)=1$; (iii) $k\left(\lambda_{1}, \lambda_{2}\right)$ $=\left(\lambda_{1}-\lambda_{2}+1\right) \lambda_{2}$ if $\lambda_{2}>0$; (iv) $k\left(\lambda_{1}, 1,1, \lambda_{4}\right)=\left(\lambda_{1}+1\right)\left(2-\lambda_{4}\right)-1$; (v) $k\left(\lambda_{1}, \lambda_{2}\right.$, $\left.\lambda_{3}, \lambda_{4}\right)=\left(\lambda_{1}-\lambda_{2}+1\right)\left(\lambda_{2}-\lambda_{3}+1\right)\left(\lambda_{3}-\lambda_{4}+1\right)$ in all other cases. 
Razmyslov [52] has proved that $\operatorname{var} M_{2}(K)$ can be defined by a system of 9 polynomial identities of degree $\leqslant 6$. In [9] this system has been reduced to 4 identities. Using the fact that Razmyslov's basis for $\operatorname{var} M_{2}(K)$ consists of polynomial identities of degree $\leqslant 6$ (but not the explicit form of the identities) and Theorem 3.4.1, computations in $B A_{m}^{(5)}$ and $B A_{m}^{(6)}$. have given a minimal generating set for $T\left(M_{2}(K)\right)$.

3.4.3. THEOREM [11]. The polynomials $\left[\left[x_{1}, x_{2}\right]^{2}, x_{1}\right]$ and $S_{4}\left(x_{1}, x_{2}, x_{3}, x_{4}\right)$ from the free associative algebra form a basis for the identities of $M_{2}(K)$.

Extensively studied objects in ring theory are generic matrix rings and some related rings. Let $\Omega=K\left[\xi_{i j}^{(r)} \mid i, j=1, \ldots, k, r=1,2, \ldots\right]$ be the polynomial algebra in $\xi_{i j}^{(r)}$. The algebra of $k \times k$ generic matrices $R_{k}(Y)$ is the unitary subalgebra of $M_{k}(\Omega)$ generated by the matrices $Y=\left\{y_{r}=\left(\xi_{i j}^{(r)}\right) \mid r=1,2, \ldots\right\}$. It is easy to see that $R_{k}(Y) \cong F^{*}\left(\operatorname{var} M_{k}(K)\right)$. The trace ring $\tilde{R}_{k}(Y)$ is generated by $R_{k}(Y)$ and all the traces from $R_{k}(Y)$. The description of $\tilde{R}_{k}\left(y_{1}, \ldots, y_{m}\right)$ as a GL ${ }_{m}$-module is given in [26] and explicit computations with $\tilde{R}_{2}(Y)$ have been done in several papers $([26,51]$ etc.). Here we combine the exposition of [51] with the approach of Section 3.1.

3.4.4. TheOREM [51]. Let $\tilde{R}_{2}(Y)$ be the $2 \times 2$ trace ring. Then:

(i) The following $K$-algebra isomorphism holds:

$$
\left.\cdot \tilde{R}_{2}(Y) \cong K\left[z_{1}, z_{2}, \ldots\right]\right]^{\#} \otimes_{K} K\langle X\rangle^{\#} / U,
$$

where $U=\left\{f\left(x_{1}, \ldots, x_{m}\right) \in K\langle X\rangle^{\#} \mid f\left(b_{1}, \ldots, b_{m}\right)=0\right.$ for all $b_{1}, \ldots, b_{m}$ $\left.\dot{\epsilon} \mathrm{sl}_{2} \subset M_{2}(K)\right\}$.

(ii) $K\left\langle x_{1}, \ldots, x_{m}\right\rangle^{*} /\left(K\left\langle x_{1}, \ldots, x_{m}\right\rangle^{*} \cap U\right)=\sum N_{m}\left(\lambda_{1}, \lambda_{2}, \lambda_{3}\right)$, where the summation is over all partitions $\lambda=\left(\lambda_{1}, \lambda_{2}, \lambda_{3}\right)$.

(iii) As a. $\mathrm{GL}_{m}$-module, the centre of $\tilde{R}_{2}\left(y_{1}, \ldots, y_{m}\right)$ is isomorphic to $K\left[z_{1}, \ldots, z_{m}\right]^{\#} \otimes_{K} \sum N_{m}\left(2 \mu_{1}+\lambda_{3}, 2 \mu_{2}+\lambda_{3}, \lambda_{3}\right)$.

Sketch of proof. (i) The Cayley-Hamilton theorem yields that for a $2 \times 2$ matrix $u$,

$$
u^{2}-(\operatorname{tr} u) u+\left(\operatorname{tr}^{2} u-\operatorname{tr} u^{2}\right) / 2=0,
$$

hence $\operatorname{tr} u^{2}=2 u^{2}-2(\operatorname{tr} u) u+\operatorname{tr}^{2} u$. Since $\operatorname{tr} u v=\operatorname{tr} v u$, the linearization of this equation gives

$$
\operatorname{tr} u v=u v+v u-(\operatorname{tr} u) v-(\operatorname{tr} v) u+(\operatorname{tr} u)(\operatorname{tr} v) .
$$

Therefore, $\tilde{R}_{,}(Y)$ is generated by $y_{i}$ and $\operatorname{tr} y_{i}, i=1,2, \ldots$ But $y_{i}=\operatorname{tr} y_{i} / 2+y_{i}^{0}$, where $y_{i}^{0}$ is a matrix with zero trace, i.e.

$$
\begin{aligned}
\tilde{R}_{2}(Y)=K\left\langle y_{i}^{0}, \operatorname{tr} y_{i}\right| i & =1,2, \ldots\rangle^{\#} \\
& \cong K\left[\operatorname{tr} y_{i} \mid i=1,2, \ldots\right]^{*} \otimes_{K} K\left\langle y_{i}^{0} \mid i=1,2, \ldots\right\rangle^{\#} .
\end{aligned}
$$


Since the traces of $y_{i}$ are algebraically independent, $K\left[\operatorname{tr} y_{i} \mid i=1,2, \ldots\right]^{\#} \cong$ $K\left[z_{1}, z_{2}, \ldots\right]^{*}$. On the other hand, it is easy to prove that $K\left\langle y_{i}^{0} \mid i=1,2, \ldots\right\rangle^{\#} \cong K\langle X\rangle^{*} / U$, where $U$ is the set of all weak polynomial identities of the pair $\left(M_{2}(K), \mathrm{sl}_{2}\right)$ (see [52] for details), i.e.

$$
U=\left\{f\left(x_{1}, \ldots, x_{m}\right) \in K\langle X\rangle^{\#} \mid f\left(b_{1}, \ldots, b_{m}\right)=0 \text { for all } b_{i} \in \mathrm{sl}_{2} \subset M_{2}(K)\right\} .
$$

(ii) Repeating verbatim the arguments of the proof of Theorem 3.3.1 and Corollary 3.3.2 we see that the standard generator $f_{\lambda}\left(x_{1}, x_{2}, x_{3}\right)$ of $A_{m} /\left(A_{m} \cap U\right)$ is a weak identity for the pair $\left(M_{2}(K), \mathrm{sl}_{2}\right)$ if and only if $f_{\lambda}\left(a_{1}, a_{2}, a_{3}\right)=0, a_{1}, a_{2}, a_{3}$ being defined in (4). It follows that $A_{m} /\left(A_{m} \cap U\right) \subset \sum N_{m}\left(\lambda_{1}, \lambda_{2}, \lambda_{3}\right)$. Since

$$
g_{\lambda}\left(x_{1}, x_{2}, x_{3}\right)=S_{3}^{\lambda_{3}}\left(x_{1}, x_{2}, x_{3}\right) S_{2}^{\lambda_{2}-\lambda_{3}}\left(x_{1}, x_{2}\right) x_{1}^{\lambda_{1}-\lambda_{2}}
$$

is a standard generator for $N_{m}\left(\lambda_{1}, \lambda_{2}, \lambda_{3}\right)$ and $g_{\lambda}\left(a_{1}, a_{2}, a_{3}\right) \neq 0$, all $N_{m}\left(\lambda_{1}, \lambda_{2}, \lambda_{3}\right)$ do enter the decomposition of $K\left\langle y_{1}^{0}, \ldots, y_{m}^{0}\right\rangle^{*}$ and this gives the desired result.

(iii) The proof makes use of the fact that, in the notation of (ii), $g_{\lambda}\left(a_{1}, a_{2}, a_{3}\right)$ is a scalar matrix if and only if $\lambda_{1}-\lambda_{2}$ and $\lambda_{2}-\lambda_{3}$ are even integers.

\subsection{The Jordan algebra of a symmetric bilinear form}

Let $V_{p}$ be a vector space of dimension $p$ with a nondegenerate symmetric bilinear form $\langle$,$\rangle . Then G_{p}=K+V_{p}$ has a structure of a Jordan algebra with multiplication

$$
(\alpha+v)(\beta+w)=(\alpha \beta+\langle v, w\rangle)+(\alpha w+\beta v), \quad \alpha, \beta \in K, v, w \in V_{p} .
$$

For $p>1$, the $G_{p}$ form a family of simple Jordan algebras. We call the basis $r_{1}, \ldots, r_{p}$ of $V_{p}$ good if it can be transformed to an orthogonal basis of $V_{p}$. ror every orthogonal basis $s_{1}, \ldots, s_{p}$

$$
\left(s_{j_{1}} \ldots\right)\left(\ldots s_{j_{n}}\right)=\varepsilon s_{1}^{\delta_{1}} \ldots s_{p}^{\delta_{p}},
$$

$\varepsilon \in K, \delta_{i}=0,1$ and $\delta_{i}$ has the same parity as $\operatorname{deg}_{s_{i}}\left(s_{j_{1}} \ldots\right)\left(\ldots s_{j_{n}}\right)$. Since the good bases are dense in $V_{p}^{p}$, Theorem 3.1.5 allows us to establish the following result.

3.5.1. TheOREM [16]. (i) $B_{m}\left(\operatorname{var} G_{p}\right)=K+\sum N_{m}\left(\lambda_{1}, \ldots, \lambda_{p}\right)$, where the summation runs over all partitions $\left(\lambda_{1}, \ldots, \lambda_{p}\right)$ such that $\lambda_{2}>0$ and at most one of the integers $\lambda_{i}$ is odd.

(ii) $B_{m}\left(\operatorname{var} G_{\infty}\right)=\left[\left(\sum_{n \geqslant n} N_{m}(2)^{\otimes} \otimes^{\circledR}\right) \otimes_{K}\left(K+N_{m}(1)\right)\right] / \sum_{n \geqslant 1} N_{m}(n)$.

(Compare this result with (2)!) 


\section{Codimensions and Hilbert series}

\subsection{Reductions}

Let $N_{m}^{\prime}$ and $N_{m}^{\prime \prime}$ be $\mathrm{GL}_{m}$-submodules of $F_{m}$ with $N_{m}^{\prime} \subset F_{m}^{(p)}, N_{m}^{\prime \prime} \subset F_{m}^{(q)}$, $N_{m}^{\prime} \otimes_{K} N_{m}^{\prime \prime} \subset F_{m}^{(p+q)}$. Assuming $m \geqslant p+q$, define $M^{\prime}=P_{p} \cap N_{m}^{\prime}, M_{m}^{\prime \prime}=P_{q} \cap N_{m}^{\prime \prime}$, $M=P_{p+q} \cap N_{m}^{\prime} \otimes_{K} N_{m}^{\prime \prime}$. Then the formulas

$$
\begin{aligned}
& H\left(N_{m}^{\prime} \otimes_{K} N_{m}^{\prime \prime}, t_{1}, \ldots, t_{m}\right)=H\left(N_{m}^{\prime}, t_{1}, \ldots, t_{m}\right) H\left(N_{m}^{\prime \prime}, t_{1}, \ldots, t_{m}\right), \\
& \operatorname{dim} M=\operatorname{dim}\left(\left(M^{\prime} \otimes_{K} M^{\prime \prime}\right) \uparrow \operatorname{Sym}(p+q)\right)=\operatorname{dim} M^{\prime} \cdot \operatorname{dim} M^{\prime \prime}\left(\begin{array}{c}
P_{p}^{+q} \\
p
\end{array}\right)
\end{aligned}
$$

simplify the computing of the Hilbert series of $F_{m}(U)$ and the codimensions of $U$. Theorem 2.2.1 (iii) reduces the computing of the Hilbert series of $F_{m}(\boldsymbol{U})$ to that of $B_{m}(U)$. A similar result holds for the codimensions.

4.1.1. THEOREM $[13,16,17]$. Let $\boldsymbol{U}$ be a variety of unitary algebras and let $\gamma_{n}(\boldsymbol{U})=\operatorname{dim} \Gamma_{n}(\boldsymbol{U}), n=0,2,3, \ldots$, be the sequence of the proper codimensions of $\boldsymbol{U}$. Then $c_{n}(\boldsymbol{U}), \gamma_{n}(\boldsymbol{U}) ; c(\boldsymbol{U}, t)=\sum c_{n}(\boldsymbol{U}) t^{n}, \gamma(\boldsymbol{U}, t)=\sum \gamma_{n}(\boldsymbol{U}) t^{n} ; \tilde{c}(\boldsymbol{U}, t)=$ $\sum c_{n}(\boldsymbol{U}) t^{n} / n !, \tilde{\gamma}(\boldsymbol{U}, t)=\sum \gamma_{n}(\boldsymbol{U}) t^{n} / n !$ are related by the following equalities:

(i) $c_{n}(\boldsymbol{U})=\sum_{s=0}^{n}\left(\begin{array}{c}n \\ 0\end{array}\right) \gamma_{s}(\boldsymbol{U})$

(ii) $c(\boldsymbol{U}, t)=\gamma(\boldsymbol{U}, t /(1-t)) /(1-t)$;

(iii) $\tilde{c}(\boldsymbol{U}, t)=e^{t} \tilde{\gamma}(\boldsymbol{U}, t)$.

\subsection{Grassmann, matrix and related algebras}

The codimension of the Grassmann algebra have been computed in [37]. Here we give an alternative proof.

4.2.1. THEOREM [37]. (i) $c_{n}(E)=2^{n-1}, n \geqslant 1$;

(ii) $c(\operatorname{var} E, t)=1 / 2+1 /(2(1-2 t))$;

(iii) $\tilde{c}(\operatorname{var} E, t)=1 / 2+e^{2 t} / 2$.

Proof. In virtue of Theorem 3.2.1, $\Gamma_{n}(\operatorname{var} E)=M\left(1^{n}\right)$ for $n$ even and $\Gamma_{n}(\operatorname{var} E)=0 \quad$ for $n$ odd. Since $\operatorname{dim} M\left(1^{n}\right)=1$, we obtain $\gamma(\operatorname{var} E, t)$ $=1+t^{2}+t^{4}+\ldots=1 /\left(1-t^{2}\right)$ and $\tilde{\gamma}(\operatorname{var} E, t)=\left(e^{t}+e^{-t}\right) / 2$ and the result follows immediately from Theorem 4.1.1.

The computing of the codimensions of the $k \times k$ matrices seems to be a very difficult problem. The asymptotic behaviour of $c_{n}\left(M_{k}(K)\right)$ has been established in a series of papers by Regev (see $[25,56]$ for references). Up till now only for $2 \times 2$ matrices has an explicit formula been obtained.

4.2.2. THEOREM [51]. (i) $c\left(\operatorname{var} M_{2}(K), t\right)=\left(1-2 t-(1-4 t)^{1 / 2}\right) /\left(2 t^{2}\right)-t^{3} /(1-t)^{4}$ $+1 /(1-t)-1 /(1-2 t)$

(ii) $c_{n}\left(M_{2}(K)\right)=\left(\begin{array}{c}2 n+2 \\ n+1\end{array}\right) /(n+2)-\left(\begin{array}{c}n \\ 3\end{array}\right)+1-2^{n}$;

(iii) [56] $c_{n}\left(M_{2}(K)\right)$ equals asymptotically $4^{n+1} /\left(n(\pi n)^{1 / 2}\right)$. 
Proof. We follow the exposition of [19].

(i) In virtue of Theorem 3.4.1 it suffices to obtain an explicit formula for $g(t)=\sum \operatorname{dim} M\left(\lambda_{1}, \lambda_{2}, \lambda_{3}\right) t^{\lambda_{1}+\lambda_{2}+\lambda_{3}}$. By the Littlewood-Richardson rule,

$$
\sum N_{m}(p, p) \otimes_{K} \sum N_{m}(q) \cong \sum N_{m}\left(\lambda_{1}, \lambda_{2}, \lambda_{3}\right)
$$

and hence, applying (6) as in Theorem 4.1.1 (ii), $g(t)=h(t /(1-t)) /(1-t)$, where $h(t)=\sum \operatorname{dim} M(p, p) t^{2 p}$. Applying the hook formula for $\operatorname{dim} M(p, p)$ we get $\operatorname{dim} M(p, p)=(2 p) ! /(p !(p+1) !)=\left(\begin{array}{c}2 p \\ p\end{array}\right) /(p+1)$ and some calculations show that $h(t)=\left(1-\left(1-4 t^{2}\right)^{1 / 2}\right) /\left(2 t^{2}\right), g(t)=\left(1-t-\left(1-2 t-3 t^{2}\right)^{1 / 2}\right) /\left(2 t^{2}\right)$. Finally, we obtain the expression for $c\left(\operatorname{var} M_{2}(K), t\right)$. The assertions (ii) and (iii) are consequences of (i).

Similar considerations allow one to compute the Hilbert series of $F_{m}\left(\operatorname{var} M_{2}(K)\right)$. For $m=2$ this has been done in [29] (see also [13]). The general case has been handled in [26] (see also [13,39]). Here we prove the case $m=2$ only.

\subsubsection{THEOREM.}

$H\left(\operatorname{var} M_{2}(K), t_{1}, t_{2}\right)=\left(1-t_{1}\right)^{-1}\left(1-t_{2}\right)^{-1}\left(1+t_{1} t_{2}\left(1-t_{1} t_{2}\right)^{-1}\left(1-t_{1}\right)^{-1}\left(1-t_{2}\right)^{-1}\right)$.

Proof. We make use of Theorem 3.4.1, the Littlewood-Richardson rule and (5):

$$
\begin{aligned}
H\left(B_{2}\left(\operatorname{var} M_{2}(K)\right), t_{1}, t_{2}\right)=H\left(\sum N_{2}\left(\lambda_{1}, \lambda_{2}\right)-\sum_{n \geqslant 1} N_{2}(n), t_{1}, t_{2}\right) \\
=H\left(\sum N_{2}(p, p) \otimes_{K} \sum N_{2}(q)+K-K\left[x_{1}, x_{2}\right]^{\#}, t_{1}, t_{2}\right) \\
=\left(H\left(\sum N_{2}(p, p), t_{1}, t_{2}\right)-1\right) H\left(K\left[x_{1}, x_{2}\right]^{\#}, t_{1}, t_{2}\right)+1 \\
=\left(\sum\left(t_{1} t_{2}\right)^{p}-1\right)\left(1-t_{1}\right)^{-1}\left(1-t_{2}\right)^{-1}+1 \\
=1+t_{1} t_{2}\left(1-t_{1} t_{2}\right)^{-1}\left(1-t_{1}\right)^{-1}\left(1-t_{2}\right)^{-1}
\end{aligned}
$$

and Theorem 2.2.1 (iii) gives the desired result. $[3,13]$.

In the same manner one can express the Hilbert series $\dot{H}\left(\operatorname{varsl}_{2}, t_{1}, t_{2}\right)$

Developing a complicated technique, including combinatorial methods and analysis (e.g. evaluation of multiple integrals) Regev $([55,56]$ and the references there) has established the asymptotic behaviour of the codimensions of the $k \wedge k$ matrices and of some related algebras. In particular, $c_{n}\left(M_{k}(K)\right)$ equals asymptotically

$$
(2 \pi)^{(1-k) / 2} 2^{\left(1-k^{2}\right) / 2} 1 ! \ldots(k-1) ! k^{\left(k^{2}+4\right) / 2} n^{\left(1-k^{2}\right) / 2} k^{2 n+2}
$$

(there are monsters not only in the theory of finite simple groups).

Many interesting results have been obtained on the module structure and the Hilbert series of the trace ring $\tilde{R}_{k}(Y)$ and its centre $\tilde{C}_{k}(Y)$. It turns out that 
as a commutative algebra $\widetilde{C}_{k}(Y)$ enjoys a series of interesting properties [28]. In particular,

4.2.4. TheOREM ([39] for $k=2$, $[28,61]) . H\left(\tilde{C}_{k}\left(y_{1}, \ldots, y_{m}\right), t_{1}^{-1}, \ldots, t_{m}^{-1}\right)$ $=(-1)^{d}\left(t_{1} \ldots t_{m}\right)^{k^{2}} H\left(\tilde{C}_{k}\left(y_{1}, \ldots, y_{m}\right), t_{1}, \ldots, t_{m}\right)$, where $d$ is the Krull dimension of $\tilde{C}_{k}\left(y_{1}, \ldots, y_{m}\right)$. A similar functional equation holds for $\tilde{R}_{k}\left(y_{1}, \ldots, y_{m}\right)$.

Formanek's proof is based on the investigations of the invariants of $k \times k$ matrices [26]; Teranishi's approach is completely different and applies the Cauchy integral formula to the Molien-Weyl expression for the Hilbert series as a multiple integral. As a consequence Teranishi has evaluated $H\left(\tilde{C}_{k}\left(y_{1}, y_{2}\right), t_{1}, y_{2}\right)$ for $k=3,4[61,62]$.

Kemer [34] has developed the structure theory of the T-ideals in the free associative algebra. An important role in his approach is played by the matrix algebras with entries from the Grassmann algebra. The simplest example is the subalgebra $M_{11}=\left[\begin{array}{ll}E^{0} & E^{1} \\ E^{1} & E^{0}\end{array}\right]$ of $M_{2}(E)$ consisting of all $2 \times 2$ matrices $\left(a_{i j}\right)$, $i, j=1,2$, such that $a_{11}, a_{22} \in E^{0}, a_{12}, a_{21} \in E^{1}$ in the canonical grading $E=E^{0}+E^{1}$ (see the proof of Theorem 3.2.1). The polynomial identities of $M_{11}$ are the same as those of the tensor square $E \otimes_{K} E$. It turns out that in some sense the properties of $E \otimes_{K} E$ are similar to those of $M_{2}(K)$. The quantitative results on the polynomial identities of $E \otimes_{K} E$ obtained by Popov [47] have allowed him to find also a basis for the T-ideal $T\left(E \otimes_{K} E\right)$.

4.2.5. THEOREM [47]. (i) $\Gamma_{n}\left(\operatorname{var} E \otimes_{K} E\right)=\sum M\left(\lambda_{1}, 2^{p}, 1^{q}\right), n>0$, where the summation is over all $\lambda_{1}, p, q$ such that $\lambda_{1}+2 p+q=n$ and $\left(\lambda_{1}, 2^{p}, 1^{q}\right) \neq(n),\left(1^{2 k+1}\right)$.

(ii) The polynomial identities $\left[\left[x_{1}, x_{2}\right],\left[x_{3}, x_{4}\right], x_{5}\right]$ and $\left[\left[x_{1}, x_{2}\right]^{2}, x_{1}\right]$ form a basis of the T-ideal $T\left(E \otimes_{K} E\right) \triangleleft K\langle X\rangle^{\#}$.

An approach similar to that of Theorem 4.2.2 gives the explicit formula for the codimensions of $E \otimes_{\mathrm{K}} E$.

4.2.6. THEOREM [19]. (i) $c\left(\operatorname{var} E \otimes_{\mathrm{K}} E, t\right)=1 / 2+1 /\left(2(1-4 t)^{1 / 2}\right)$ $+t /(1-t)^{2}+1 /(1-t)-1 /(1-2 t)$

(ii) $c_{n}\left(E \otimes_{K} E\right)=\left(\begin{array}{c}2 n \\ n\end{array}\right) / 2+n+1-2^{n}, n>0$;

(iii) $c_{n}\left(E \otimes_{K} E\right)$ equals asymptotically $4^{n} /\left(2(\pi n)^{1 / 2}\right)$.

Sketch of proof. It suffices to prove (i) only; then (ii) and (iii) follow immediately. The Littlewood-Richardson rule gives

$$
\begin{aligned}
\sum N_{m}\left(2^{2 p}, 1^{2 q}\right) \otimes_{K} \sum N_{m}(n) & \cong \sum N_{m}\left(\lambda_{1}, 2^{p}, 1^{q}\right) \\
& \cong B_{m}\left(\operatorname{var} E \otimes_{K} E\right)+\sum_{n>0} N_{m}(n)+\sum_{k>0} N_{m}\left(1^{2 k+1}\right) .
\end{aligned}
$$


As in the proof of Theorem 4.2.2, explicit computations show that

$$
g_{1}(t)=\sum \operatorname{dim} M\left(\lambda_{1}, 2^{p}, 1^{q}\right) t^{\lambda_{1}+2 p+q}=h_{1}(t /(1-t)) /(1-t),
$$

where $h_{1}(t)=\sum \operatorname{dim} M\left(2^{2 p}, 1^{2 q}\right) t^{4 p+2 q}$. The application of the hook formula gives $h_{1}(t)=\left(1+\left(1-4 t^{2}\right)^{1 / 2}\right) / 2$. Then the proof is completed as in Theorem 4.2.2.

There exist T-ideals of $K\langle X\rangle$ generated by important polynomial identities and which are very close to the $T$-ideals $T\left(M_{2}(K)\right)$ and $T\left(E \otimes_{K} E\right)$. These identities are the standard identity $S_{4}\left(x_{1}, x_{2}, x_{3}, x_{4}\right)$, the central-by-metabelian identity $\left[\left[x_{1}, x_{2}\right],\left[x_{3}, x_{4}\right], x_{5}\right]$ and the Hall identity $\left[\left[x_{1}, x_{2}\right]^{2}, x_{3}\right]$ handled by Kemer [33], Popov [48] and Nikolaev [44], respectively. Since these three polynomials belong to $\Gamma A_{n}, n=4,5$, without loss of generality we consider varieties of unitary algebras.

4.2.7. ThEOREM. Let $S, C$ and $H$ be the T-ideals of $K\langle X\rangle^{\#}$ generated by $S_{4}\left(x_{1}, x_{2}, x_{3}, x_{4}\right),\left[\left[x_{1}, x_{2}\right],\left[x_{3}, x_{4}\right], x_{5}\right]$ and $\left[\left[x_{1}, x_{2}\right]^{2}, x_{3}\right]$, respectively. Then:

(i) [33] $\left(B A_{m} \cap S\right) /\left(B A_{m} \cap T\left(M_{2}(K)\right)\right) \cong N_{m}(3,2)+N_{m}\left(3^{2}\right)$;

(ii) [48] $\left(B A_{m} \cap C\right) /\left(B A_{m} \cap T\left(E \otimes_{K} E\right)\right) \cong N_{m}(3,2)+N_{m}\left(3^{2}\right)$;

(iii) [44] $\left(B A_{m} \cap H\right) /\left(B A_{m} \cap T\left(M_{2}(K)\right)\right) \cong N_{m}\left(3,1^{2}\right)+2 N_{m}\left(2,1^{3}\right)+N_{m}\left(3,1^{3}\right)$ $+2 N_{m}\left(2^{2}, 1^{2}\right)+N_{m}\left(2,1^{4}\right)+N_{m}\left(2^{2}, 1^{3}\right)+\sum_{k>1} N_{m}\left(1^{2 k}\right)$.

By the way, in the proofs of (ii) and (iii) the authors have used the decomposition of the $\operatorname{Sym}(n)$-modules $\Gamma A_{n}$ for $n \leqslant 8$. As an immediate consequence of Theorems 4.2 .7 and 4.1 .1 we obtain

4.2.8. Corollary [19]. (i) $c_{n}(S)=c_{n}\left(M_{2}(K)\right)+5\left(\begin{array}{c}n \\ 5\end{array}\right)+5\left(\begin{array}{c}n \\ 6\end{array}\right)$;

(ii) $c_{n}(C)=c_{n}\left(E \otimes_{K} E\right)+5\left(\begin{array}{c}n \\ 5\end{array}\right)+5\left(\begin{array}{c}n \\ 6\end{array}\right)$;

(iii) $c_{n}(H)=c_{n}\left(M_{2}(K)\right)+2^{n-1}-1-\left(\begin{array}{c}n \\ 2\end{array}\right)+14\left(\begin{array}{c}n \\ 5\end{array}\right)+33\left(\begin{array}{c}n \\ 6\end{array}\right)+14\left(\begin{array}{c}n \\ 7\end{array}\right), n>0$.

The description of $B_{m}\left(\operatorname{var} G_{\infty}\right)$ for the variety of Jordan algebras generated by $G_{\infty}$ (see Theorem 3.5.1) gives an interesting formula for the codimensions of $G_{\infty}$ and $G_{p}$. It seems very intriguing that there exists a connection between the asymptotic behaviour of $c_{n}\left(G_{\infty}\right)$ and the Hermite polynomials (see [16] for details).

4.2.9. THEOREM [16]. (i) $c\left(\operatorname{var} G_{\infty}, t\right)=(1+t) \exp \left(t+t^{2} / 2\right)+e^{t}-e^{2 t}$; $\mathcal{c}_{n}\left(G_{\infty}\right)=g^{(n+1)}(1)+1-2^{n}$, where $g(t)=\exp \left(\left(t^{2}-1\right) / 2\right)$;

(ii) $c_{n}\left(G_{\infty}\right)=O\left((c n)^{n / 2}\right)$, where $c=\exp (\pi-1)$;

(iii) $\lim _{n \rightarrow \infty}\left(c_{n}\left(G_{p}\right)\right)^{1 / n}=p+1$;

(iv) $[18] c\left(\operatorname{var} G_{2}, t\right)=\left(t-1+(t+1) /\left(1-4 t^{2}\right)^{1 / 2}\right) /(2 t)-t /(1-t)$.

\subsection{Products of T-ideals}

In this section we deal with unitary associative algebras only. For two algebras $P$ and $Q$, let $U=T(P)$ and $V=T(Q)$ be the corresponding T-ideals of 
$A^{\#}=K\langle X\rangle^{\#}$. For a $(P, Q)$-bimodule $M$, it is easy to see that $R=\left[\begin{array}{cc}P & M \\ O & Q\end{array}\right]$ is a $K$-algebra and $T(R) \supset W=U V$. Under some additional conditions (see [40, 1, 64]), $T(R)=W$. For example [40] this holds if $P=F^{\#}(U), Q=F^{\#}(V)$, $M=S /(U S+S V)$, where $S=\sum_{i} A^{\#} y_{i} A^{\#}$ is a free $\left(A^{\#}, A^{\#}\right)$-bimodule of countable rank. Another example is the algebra $U T_{p}(K)$ of all $p \times p$ upper-triangular matrices when $T\left(U T_{p}(K)\right)=(T(K))^{p}=\left[A^{\#}, A^{\#}\right]^{p}[42]$.

We discuss the following problem. How to calculate the numerical invariants of the $T$-ideal $W=U V$ if we know those of $U$ and $V$ ? The following results give the connection between the Hilbert series of the relatively free algebras of $\boldsymbol{U}, \boldsymbol{V}$ and $\boldsymbol{W}$ and their exponential codimension series.

4.3.1. TheOREM [27]. Let $V, U$ be T-ideals of $A_{m}^{\#}=K\left\langle x_{1}, \ldots, x_{m}\right\rangle^{\#}$ and let $W=U V$. Then

$$
\begin{aligned}
H\left(\boldsymbol{W}, t_{1}, \ldots, t_{m}\right)= & H\left(\boldsymbol{U}, t_{1}, \ldots, t_{m}\right)+H\left(V, t, \ldots, t_{m}\right) \\
& +\left(t_{1}+\ldots+t_{m}-1\right) H\left(U, t_{1}, \ldots, t_{m}\right) H\left(V, t_{1}, \ldots, t_{m}\right) .
\end{aligned}
$$

Proof (see e.g. [17]). Since $F_{m}^{\#}(\boldsymbol{U})=A_{m}^{\#} / U$ and $H\left(F_{m}^{\#}(U), t_{1}, \ldots, t_{m}\right)+H\left(U, t_{1}, \ldots, t_{m}\right)=H\left(A_{m}^{\#}, t_{1}, \ldots, t_{m}\right)=1 /\left(1-\left(t_{1}+\ldots+t_{m}\right)\right)$, the equality (7) is equivalent to

$$
H\left(U, t_{1}, \ldots, t_{m}\right) H\left(V, t_{1}, \ldots, t_{m}\right)=H\left(U V, t_{1}, \ldots, t_{m}\right) H\left(A_{m}^{\#}, t_{1}, \ldots, t_{m}\right) .
$$

The free algebra $A_{m}^{\#}$ is a FIR-ring and every homogeneous ideal of $A_{m}^{\#}$ has a free system of homogeneous generators as a left (or right) $A_{m}^{\#}$-module. Hence there exist multihomogeneous polynomials $u_{1}, u_{2}, \ldots$ and $v_{1}, v_{2}, \ldots$ such that $U=\sum u_{i} A_{m}^{\#}, V=\sum A_{m}^{\#} v_{j}$ and the sums are direct. Therefore

$$
H\left(U, t_{1}, \ldots, t_{m}\right)=\sum t^{\left|u_{i}\right|} H\left(A_{m}^{*}, t_{1}, \ldots, t_{m}\right),
$$

where $t^{\left|u_{i}\right|}=t_{1}^{p_{1}} \ldots t_{m}^{p_{m}}, p_{s}=\operatorname{deg}_{x_{s}} u_{i}$, and similarly for $H\left(V, t_{1}, \ldots, t_{m}\right)$. Since $U V=\sum u_{i}\left(A_{m}^{\#}\right)^{2} v_{j}$ and $\left(A_{m}^{\#}\right)^{2}=A_{m}^{\#}$, it follows that $U V=\sum u_{i} A_{m}^{\#} v_{j}$, $H\left(U V, t_{1}, \ldots, t_{m}\right)=\sum t^{\left|u_{i}\right|} t^{\left|v_{j}\right|} H\left(A_{m}^{\#}, t_{1}, \ldots, t_{m}\right)$ and this gives the desired result.

4.3.2. THEOREM [17]. The exponential codimension series of $U, V$ and $W=U V$ satisfy the equation

$$
\tilde{c}(\boldsymbol{W}, t)=\tilde{c}(\boldsymbol{U}, t)+\tilde{c}(V, t)+(t-1) \tilde{c}(\boldsymbol{U}, t) \tilde{c}(\boldsymbol{V}, t) .
$$

Proof. Since $c_{n}(W)$ equals the coefficient of $t_{1} \ldots t_{n}$ in $H\left(A_{n}^{\#} \cap W, t_{1}, \ldots, t_{n}\right)$, Theorem 4.3.1 gives

$$
\begin{aligned}
c_{n}(W)= & c_{n}(U)+c_{n}(V)+\sum_{k}(n ! /(n ! k !(n-k-1) !)) c_{k}(U) c_{n-k-1}(V) \\
& -\sum_{k}(n ! /(k !(n-k) !)) c_{k}(U) c_{n-k}(V) .
\end{aligned}
$$


Hence

$$
\tilde{c}(\boldsymbol{W}, t)=\tilde{c}(\boldsymbol{U}, t)+\tilde{c}(\boldsymbol{V}, t)+(t-1) \sum_{n} \sum_{k} c_{k}(\boldsymbol{U}) c_{n-k}(V) t^{n} /(k !(n-k) !)
$$

and this completes the proof because

$$
\sum_{n} \sum_{k} c_{k}(U) c_{n-k}(V) t^{n} /(k !(n-k) !)=\tilde{c}(U, t) \tilde{c}(V, t) .
$$

The simplest application of Theorem 4.3.2 is when $W_{p}=U^{p}$ and $U$ is the commutator ideal of $A^{\#}$, i.e. $U=\operatorname{var} K$. In this case the standard notation for $W_{p}$ is $N_{p} A, W_{p}$ is defined by the identity $\left[x_{1}, x_{2}\right] \ldots\left[x_{2 p-1}, x_{2 p}\right]$ and this variety enjoys many interesting properties [38]. Since $c_{n}(U)=1$ and $\tilde{c}(U, t)=e^{t}$, it is easy to establish the following consequence of Theorem 4.3.2.

4.3.3. Corollary [17]. $\tilde{c}\left(N_{p} A, t\right)=e^{t} \sum_{k=0}^{-1}\left(1+(t-1) e^{t}\right)^{k}$.

Similarly, for the T-ideal $U=T(E)$ Theorem 4.2 .1 gives

4.3.4. Corollary [17]. For the variety $V_{p}=N_{p}$ var $E$ with a T-ideal $V_{p}=(T(E))^{p}$ generated by $\left[x_{1}, x_{2}, x_{3}\right] \ldots\left[x_{3 p-2}, x_{3 p-1}, x_{3 p}\right]$

$$
c\left(V_{p}, t\right)=\sum_{k=0}^{p} f_{k p}(t) e^{2 k t}
$$

where the $f_{k p}(t)$ are polynomials in $t$ and $f_{p p}(t)=(t-1)^{p-1} / 2^{p}$.

Very often the asymptotic behaviour of the codimension sequence of a T-ideal is more important than the explicit formula. For example, for an associative PI-algebra $R$ Regev [54] has established that $c_{n}(R) \leqslant d^{n}$ for a suitable $d$ and this has allowed him to show that a tensor product of PI-algebras is PI again. Another application is due to Kemer [32] who has used the asymptotic behaviour of the cocharacter sequence to give the final form of a result of Razmyslov [53] on the nilpotency of the Jacobson radical of a linitely generated PI-algebra (see Theorem 1.5.1).

An estimate of the asymptotics of the codimension sequence $c_{n}(U)$ of a variety $U$ is $\lim \sup \left(c_{n}(U)\right)^{1 / n}$. Clearly, $1 / \lim \sup \left(c_{n}(U)\right)^{1 / n}$ equals the radius of convergence of the series $c(U, t)$. It is unknown if $\lim _{n \rightarrow \infty}\left(c_{n}(U)\right)^{1 / n}$ exists for all $U$ but in the few cases when it is explicitly calculated always $\lim _{n \rightarrow \infty}\left(c_{n}(U)\right)^{1 / n}$ does exist and is an integer. We suggest $\lim _{n \rightarrow \infty}\left(c_{n}(U)\right)^{1 / n}$ as a measure of the complexity of $\boldsymbol{U}$.

43.5. Defintion [17]. We call the variety $U$ extremal if $\lim _{n \rightarrow \infty}\left(c_{n}(U)\right)^{1 / n}$ exists and for any proper subvariety $\boldsymbol{V}$ of $\boldsymbol{U}$,

$$
\lim _{n \rightarrow \infty}\left(c_{n}(U)\right)^{1 / n}>\lim \sup \left(c_{n}(V)\right)^{1 / n}
$$

Freely restated, this means that $U$ is more complicated than its subvarieties. The most important examples of extremal varieties are the matrix varieties. 
4.3.6. THEOREM $[26,55,17]$. The varieties of associative algebras var $M_{p}(K)$ are extremal: $\lim _{n \rightarrow \infty}\left(c_{n}\left(M_{p}(K)\right)\right)^{1 / n}=p^{2}$ and for every proper subvariety $V$, $\lim \sup \left(c_{n}(V)\right)^{i / n} \leqslant p^{2}-1$.

It turns out that the varieties of Corollaries 4.3 .3 and 4.3 .4 are also extremal.

43.7. THEOREM [17]. Let $\boldsymbol{W}_{p}$ and $V_{p}$ be the varieties of associative (not necessarily unitary) algebras defined by the identities $\left[x_{1}, x_{2}\right] \ldots\left[x_{2 p-1}, x_{2 p}\right]$ and $\left[x_{1}, x_{2}, x_{3}\right] \ldots\left[x_{3 p-2}, x_{3 p-1}, x_{3 p}\right]$, respectively. Then $W_{p}$ and $V_{p}$ are extremal:

(i) $\lim _{n \rightarrow \infty}\left(c_{n}\left(W_{p}\right)\right)^{1 / n}=p, \lim _{n \rightarrow \infty}\left(c_{n}\left(V_{p}\right)\right)^{1 / n}=2 p$;

(ii) For any proper subvarieties $U_{1} \subset \boldsymbol{W}_{p}$ and $U_{2} \subset V_{p}$

$$
\lim \sup \left(c_{n}\left(U_{1}\right)\right)^{1 / n} \leqslant p-1, \quad \lim \sup \left(c_{n}\left(U_{2}\right)\right)^{1 / n} \leqslant 2 p-1 .
$$

The proof is based on a careful investigation of the cocharacter sequences of the subvarieties of $W_{p}$ and $V_{p}$. In particular, the decomposition of $P_{n}\left(W_{p}\right)$ into a sum of irreducible $\operatorname{Sym}(n)$-submodules is of the form $P_{n}\left(W_{p}\right)=\sum k(\lambda) M(\lambda)$, where $\lambda=\left(\lambda_{1}, \ldots, \lambda_{s}\right)$ and $\lambda_{p+1}$ and $s$ are bounded by constants depending on $p$ only and $\lambda_{p}$ is not bounded. On the other hand, for every proper subvariety $U_{1}$ of $W_{p}, P_{n}\left(U_{1}\right)=\sum k_{1}(\lambda) M(\lambda)$, where $\lambda_{p}$ is bounded by a constant depending on $U_{1}$. Then an estimate of the dimensions of the $\operatorname{Sym}(n)$-modules allows one to obtain $\lim \sup \left(c_{n}\left(U_{1}\right)\right)^{1 / n} \leqslant p-1$. The bound for $U_{2} \subset V_{p}$ is similar. We refer to [17] for details.

With some modifications the result for $\boldsymbol{W}_{p}$ holds for Lie algebras as well [17]. In this case $W_{p}$ has to be replaced by the variety $A^{3} \cap N_{p-1} A$ of all Lie algebras which are solvable of class 3 and satisfy the identity $\left(x_{1} x_{2}\right)$ $\ldots\left(x_{2 p-1} x_{2 p}\right)$.

Finally, we shall mention another important example of extremal varieties of Jordan algebras.

4.3.8. THEOREM [36]. Let $G_{p}$ be the Jordan algebra of a nondegenerate symmetric bilinear form on a vector space of dimension $p$. Then the varieties $\operatorname{var} G_{p}$ and $\operatorname{var} G_{\infty}$ are extremal:

(i) For a proper subvariety $U$ of $\operatorname{var} G_{p}$

$$
\lim \sup \left(c_{n}(U)\right)^{1 / n} \leqslant p<\lim _{n \rightarrow \infty}\left(c_{n}\left(G_{p}\right)\right)^{1 / n}=p+1
$$

When $U$ is a variety of unitary algebras, $\lim _{n \rightarrow \infty}\left(c_{n}(U)\right)^{1 / n}$ exists and is an integer.

(ii) For a proper subvariety $U$ of $\operatorname{var} G_{\infty}$

$$
\lim \sup \left(c_{n}(U)\right)^{1 / n}<\infty=\lim _{n \rightarrow \infty}\left(c_{n}\left(G_{\infty}\right)\right)^{1 / n}
$$




\section{References}

[1] A. Sh. Abakarov, On the identities of the algebra of triangular matrices, in: Modules and Algebraic Groups, Zap. Nauchn. Sem. LOMI 114 (1982), 7-27 (in Russian).

[2] A. Z. Anan in and A. R. Kemer, Varieties of associative algebras whose lattices of subvarieties are distributive, Sibirsk. Mat. Zh. 17 (1976), 723730 (in Russian).

[3] Yu. A. Bakhturin, 2-Variable identities of the Lie algebra sl $(2, k)$, Trudy Sem. Petrovsk. 5 (1979), 205-208 (in Russian).

[4] - Identities in Lie Algebras, Nauka, Moscow 1985 (in Russian).

[5] Yu. A. Bakhturin and V. S. Drensky, Varieties of Lie algebras, in: Algebraic Structures, Solia, to appear (in Russian).

[6] A. Berele, Homogeneous polynomial identities, Israel J. Math. 42 (1982), 258-272.

[7] A. Berele and A. Rege v, Applications of hook Young diagrams to P. I. algebras, J. Algebra 82 (1983), 559-567.

[8] A. Braun. The nilpotency of the radical in a finitely generated P.I. ring, ibid. 89 (1984), 375-396.

[9] Bu i Tuong Tri, On the basis of the identities of the matrix algebra of second order over a field of characteristic zero, Serdica 7 (1981), 187-194.

[10] V. S. Drensky, Representations of the symmetric group and varieties of linear algebras, Mat. Sb. 115 (1981), 98-115 (in Russian).

[11] - Minimal basis for the identities of a matrix algebra of second order over a field of characteristic 0, Algebra i Logika 20 (1981), 282-290 (in Russian).

[12] -, On the lattices of varieties of associative algebras, Serdica 8 (1982), 20-31 (in Russian).

[13] -, Codimensions of T-ideals and Hilbert series of relatively free algebras, J. Algebra 91 (1984), 1-17.

[14] -, T-ideals containing all matrix polynomial identities, Comm. Algebra 13 (1985), 2037-2072.

[15] -, Sur les identités polynomiales de l'algèbre de Lie des matrices d'ordre deux, C. R. Acad. Bulgare Sci. 39 (10) (1986), 17-20.

[16] -, Polynomial identities for the Jordan algebra of a symmetric bilinear form, J. Algebra 108 (1987), 66-87.

[17] -, Extremal varieties of algebras, I, II, Serdica 13 (1987), 320-332; 14 (1988), 20-27 (in Russian).

[18] -, On the identities of the three-dimensional simple Jordan algebra, Ann. Univ. Sofia Fac. Math. Méc. Livre 1 Math. 78 (1984), 57-71.

[19] -, Explicit formulas for the codimensions of some T-ideals, Sibirsk. Mat. Zh. 29 (6) (1988), 30-36 (in Russian).

[20] V. Drensky and A. Kasparian, Polynomial identities of eighth degree for $3 \times 3$ matrices, Ann. Univ. Solia Fac. Math. Méc. Livire 1 Math. 77 (1983), 175-195.

[21] V. S. Drensky and A. P. Popov, Prime varieties of associative algebras, in: Math. and Education in Math., Publ. House of Bulg. Acad. Sci., Sofia 1987, 35-52.

[22] -, -, Application of the representation theory of groups to PI-algebras, in: Algebraic Structures, Solia, to appear (in Russian).

[23] V. S. Drensky and Ts. Rashkova, Varieties of metabelian Jordan algebras, Serdica 15 (1989), to appear.

[24] V. T. Filip pov, On the variety of Mal'tsev algebras, Algebra i Logika 20 (1981), 300-314 (in Russian).

[25] E. Formanek, The polynomial identities of matrices, Contemp. Math. 13 (1982), 41-79.

[26] -, Invariants and the ring of generic matrices, J. Algebra 89 (1984), 178-223.

[27] -, Noncommutative invariant theory, Contemp. Math. 43 (1985), 87-119.

[28] -, Functional equations for character series associated with $n \times n$ matrices, Trans. Amer. Math. Soc. 294 (1986), 647--663.

[29] E. Form a nek, P. Halpin and W.-C. W. Li, The Poincaré series of the ring of $2 \times 2$ generic matrices, J. Algebra 69 (1981), 105-112. 
[30] N. Jacobson, PI-Algebras: An Introduction, Lecture Notes in Math. 441, Springer, Berlin 1975.

[31] G. James and A. Kerber, The Representation Theory of the Symmetric Group, Encyclopedia Math. Appl. 16, Addison-Wesley, Reading, Mass., 1981.

[32] A. R. Kemer, Capelli identities and nilpotency of the radical of a finitely generated PI-algebra, Dokl. Akad. Nauk SSSR 255 (1980), 793-797 (Russian).

[33] -, The ideal of identities generated by the standard identity of fourth degree, in: Proc. XVII All-Union Algebraic Conf., Vol. 1, Minsk 1983, 89-90 (in Russian).

[34] -, Varieties and $Z_{2}$-graded algebras, Izv. Akad. Nauk SSSR Ser. Mat. 48 (1984), 1042-1059 (in Russian).

[35] A. A. Klyach ko, Lie elements in the tensor algebra, Sibirsk. Mat. Zh. 15 (1974), 1296-1304 (in Russian).

[36] P. Ko oshluk ov, Polynomial identities for a family of simple Jordan algebras, Comm. Algebra 16 (1988), 1325-1371.

[37] D. Krakowsky and A. Regev, The polynomial identities of the Grassmann algebra, Trans. Amer. Math. Soc. 181 (1973), 429-438.

[38] V. N. Latyshev, Complexity of nonmatrix varieties of associative algebras, $I, I I$, Algebra i Logika 16 (1977), 149-183, 184-199 (in Russian).

[39] L. Le Bruyn, The Poincaré series of $\Pi_{m .2}$ in: Ring Theory, Proc. Antwerp 1985, Lecture Notes in Math. 1197, Springer, Berlin 1986, 90-108.

[40] J. Lewi n, A matrix representation for free associative algehras, I. II. Trans. Amer. Math. Soc. 188 (1974), 293-308, 309-317.

[41] I. G. Macdonald, Symmetric Functions and Hall Polynomials, Oxford Univ. Press (Clarendon), Oxford 1979.

[42] $\mathrm{Yu}$. N. Mal'tsev, A hasis for identities of the algehra of upper-triangular matrices, Algebra i Logika 10 (1971), 393-400 (in Russian).

[43] S. P. Mishchenko, Varieties of hypercentral-by-metabelian Lie algebras over a field of characteristic zero, Vestnik Moskov. Univ. Ser. I Mat. Mekh. 1983, No. 5, 33-37 (in Russian).

[44] R. S. Nikola ev, The structure of the T-ideal generated by the Hall identity in three variables, I, II, Serdica 13 (1987), 258-266, 361-365 (in Russian).

[45] -, Identities in three variables in the Lie algebra $\mathrm{sl}(2, K)$ over a field of characteristic zero, ibid. 14 (1988), 28-33 (in Russian).

[46] G. M. Piacentini Cattaneo, Nonassociative degree five identities not implied by commutativity: a computer upproach, in: Applied Algebra, Algebraic Algorithms and Error-Correcting Codes, Lecture Notes in Comput. Sci. 357, Springer, Berlin 1989, 336-340.

[47] A. P. Popov, Identities of the tensor square of the Grassmann algebra, Algebra i Logika 21 (1982), 442-471 (in Russian).

[48] -, On central metabelian variety of algebras, Comm. Algebra 15 (1987), 1319-1347.

[49] -, Module structure of space of proper polynomials of degree seven, C. R. Acad. Bulgare Sci. 38 (1985), 295-298.

[50] C. Procesi, The invariant theory of $n \times n$ matrices, Adv. in Math. 19 (1976), 306-381.

[51] -, Computing with $2 \times 2$ matrices, J. Algebra 87 (1984), 342-359.

[52] Yu. P. Razmyslov, Finite basing of the identities of a matrix algebra of second order over a field of characteristic 0, Algebra i Logika 12 (1973), 83-113 (in Russian).

[53] -, The Jacobson radical in Pl-algebras, ibid. 13 (1974), 337-360 (in Russian).

[54] A. Regev, Existence of identities in $A \otimes B$, Israel J. Math. 11 (1972), 131-152.

[55] -, Asymptotic values for degrees associated with strips of Young diagrams, Adv. in Math. 41 (1981), 115-136.

[56] -, On the codimension of matrix algebras, in: Algebra - Some Current Trends, Lecture Notes in Math. 1352, Springer, Berlin 1988, 162-172.

[57] L. H. Rowen, Polynomial Identities in Ring Theory, Academic Press, 1980. 
[58] A. I. Shirshov, Subalgebras of free commutative and anticommutative algebras, Mat. Sb. 34 (1954), 81-88.

[59] P. N. Siderov, Basis of identities of the algebra of triangular matrices over an arbitrary field, PLISKA Stud. Math. Bulgar. 2 (1981), 143-152 (in Russian).

[60] W. Specht, Gesetze in Ringen, I, Math. Z. 52 (1950), 557-589.

[61] Y. Teranishi, The ring of invariants of matrices, Nagoya Math. J. 104 (1986), 149-161.

[62] -, Linear diophantine equations and invariant theory of matrices, Adv. Stud. Pure Math. 11 (1987), 259-275.

[63] R. M. Thrall, On symmetrized Kronecker powers and the structure of the free Lie ring, Trans. Amer. Math. Soc. 64 (1942), 371-388.

[64] S. M. Vovsi, Triangular Products of Group Representations and Their Applications, Progr. Math. 17, Birkhäuser, Boston 1981.

[65] H. Weyl, The Classical Groups, Their Invariants and Representations, Princeton Univ. Press, Princeton, N. J., 1946. 